\title{
Successes and Challenges for Flow Control Simulations (Invited)
}

\author{
Christopher L. Rumsey* \\ NASA Langley Research Center, Hampton, VA 23681-2199, USA
}

\begin{abstract}
A survey is made of recent computations published for synthetic jet flow control cases from a CFD workshop held in 2004. The three workshop cases were originally chosen to represent different aspects of flow control physics: nominally 2-D synthetic jet into quiescent air, 3-D circular synthetic jet into turbulent boundarylayer crossflow, and nominally 2-D flow-control (both steady suction and oscillatory zero-net-mass-flow) for separation control on a simple wall-mounted aerodynamic hump shape. The purpose of this survey is to summarize the progress as related to these workshop cases, particularly noting successes and remaining challenges for computational methods. It is hoped that this summary will also by extension serve as an overview of the state-of-the-art of CFD for these types of flow-controlled flow fields in general.
\end{abstract}

\section{Introduction}

Although flow control concepts have been around a long time, the last decade or so has seen a dramatic increase in experiments and applications. Along with this increase, the need for accurate computational predictive tools has also grown. Many types of CFD methods have been applied, including low-order methods, steady or unsteady Reynoldsaveraged Navier-Stokes (RANS or URANS), large eddy simulations (LES), blended RANS-LES, and direct numerical simulations (DNS). Various degrees of success have been reported. However, most engineers today would probably agree that we are still a long way off from consistently predicting these types of flows with a high level of sustained accuracy and confidence.

In 2004 a workshop was held in Williamsburg Virginia entitled The Langley Research Center Workshop on CFD Validation of Synthetic Jets and Turbulent Separation Control (also known as "CFDVAL2004"). The experimental data and workshop results were posted to a public website. ${ }^{a}$ It is important to note that experiments can be difficult to perform for these types of unsteady flow fields. In the experiments for the workshop, an effort was made to take duplicate measurements using different techniques; this duplication highlighted the uncertainties inherent in the measurements. ${ }^{1-5}$

A summary of the workshop results can be found in Rumsey et al. ${ }^{6}$ Overall, one of the general conclusions was that CFD was only able to qualitatively predict synthetic jet flow physics, but some of this inability to achieve consistent quantitative predictions resulted from significant uncertainty regarding how to best model the unsteady boundary conditions that are always required for such flows. An important need identified was for building-block synthetic jet experiments to focus more on obtaining extremely detailed data at and near slot or orifice exits.

CFD workshops such as this are very useful because many engineers get together to try to solve common problems. With many different people, codes, and models applied to the same cases, it is usually easier to discern trends and particular deficiencies. It also often becomes clearer how to determine what future efforts are needed. CFD workshops can also be useful in bringing together CFD programmers and experimentalists. By helping to foster improved synergy between the two groups, better collaboration and improved experiments and CFD models are more likely to occur.

Another particular benefit from having many people compute the same cases is that sometimes "outliers" are easily identified, in the sense that their results lie well outside the majority (or collective). This can be useful to help workshop participants find software bugs in their CFD codes. It also helps to identify particular models or methods that are suitable/unsuitable for specific applications. These types of comparisons have proved to be very helpful at the Drag Prediction series of workshops. ${ }^{7,8}$

\footnotetext{
${ }^{*}$ Senior Research Scientist, Computational Aerosciences Branch, Mail Stop 128, Associate Fellow AIAA.

This material is declared a work of the U.S. Government and is not subject to copyright protection in the United States.2008

ahttp://cfdval2004.larc.nasa.gov [cited 6/2008].
} 
It is important to note that it is not unusual for a CFD result to agree with experimental data for the wrong reasons. It could be serendipity, cancellation of errors, or pure luck. In any case, when this happens outside of the workshop setting, such a "false positive" can often be difficult to discern, and can lead to incorrect conclusions. In the workshop setting, however, false positives are far easier to recognize for what they are. An example is shown in fig. 1. Here, results for turbulent shear stress are shown compared against the workshop hump model experimental data, with 5 different contributers performing 12 computations using different grids and different codes, all with the same turbulence model, Spalart-Allmaras (SA). ${ }^{9}$ As can be seen, all results but one are very close, giving confidence that the collective are likely using SA coded essentially the same way. The one outlier is not only very different from the others, it also happens to agree very well with the experimental data. The fact that it is an outlier means that it is computing in a different way than everyone else. Either it has a coding or user error and got lucky to agree with experiment so well, or it has happened upon a new model (by chance or on purpose) that is different from SA and better suited to model the physics. In either case, identifying outliers like this can be very beneficial when found and pursued in a spirit of scientific openness.

A brief discussion is now given about the various broad categories of methodologies brought up later in the paper. A reduced-order or low-order methodology is generally used to describe a method that involves simplifications or approximations to the typical CFD approach of solving 2-D or 3-D Navier-Stokes (Reynolds-averaged, filtered, complete, etc.) equations. For example, lumped element models, quasi-one-dimensional models, and proper orthogonal decomposition (POD) models fall in this category. Typically these simplifications are done in order to provide a less expensive way to find engineering solutions. They can be extremely useful when trying to find a viable design from among hundreds of possibilities, for example. In CFDVAL2004, there was only one low-order application among all the participants.

RANS or URANS methods make up (by far) the majority of CFD applications to flow control problems. RANS implies a steady state solution whereas URANS is unsteady, but both methods usually solve the same set of Reynoldsaveraged Navier-Stokes equations. URANS involves solution of the RANS equations in a time-accurate fashion. In the conventional decomposition for deriving the RANS equations, quantities are written as a mean plus fluctuating component: $f=\bar{f}+f^{\prime}$ and then the equations are written in terms of $\bar{f}$ (for compressible flows, mass-weighted or Favre-averaging ${ }^{10}$ is typically used, for example $\hat{f}=\overline{\rho f} / \bar{\rho}, f=\hat{f}+f^{\prime \prime}$ and the equations are written in terms of $\hat{f}$ ). Reynolds averaging is assumed to be valid in the following sense: the time scale associated with turbulent fluctuations is much smaller than the physical time step, which in turn is much smaller than the important global unsteady time scales present in the flow. ${ }^{11}$ For synthetic jet applications, there is also a well-defined forcing frequency, and the problem can be treated with the triple-decomposition proposed by Hussain and Reynolds: ${ }^{12} f=\bar{f}+\tilde{f}+f^{\prime}$, where $\bar{f}$ is the global mean, $\tilde{f}$ is the statistical contribution of the organized motion, and $f^{\prime}$ is the random component (turbulence). Because the phase-averaged value $\langle f\rangle=\bar{f}+\tilde{f}$, the decomposition can be written as $f=\langle f\rangle+f^{\prime}$. This resembles standard Reynolds splitting, and the end result is that the final conservation equations in terms of phase-averaged variables are identical in form to the standard Reynolds-averaged equations.

For RANS/URANS, the equations end up with an un-closed turbulent stress term $\tau_{i k}$ (along with un-closed heat flux for compressible flows), which must be modeled. The $\tau_{i k}$ term is typically modeled using one- or two-equation linear or nonlinear eddy viscosity turbulence models, although full Reynolds stress models that solve transport equations for each stress component are also available. In summary, RANS/URANS seeks to model the mean effects of random turbulence on the quasi-steady flow, global unsteady flow, or phase-averaged flow. As long as the assumption regarding time scales ${ }^{11}$ is valid, RANS/URANS should be a theoretically valid methodology. But the devil is in the details: how well do today's turbulence models represent the mean effects of unsteady, random turbulent eddies? As most people realize, in regard to wall-bounded flows their success is typically limited to attached or mildly separated flows. They typically do not consistently model the mean effects of turbulent eddies present in larger separated regions very well.

LES equations are derived by applying a low-pass filter, often defined as a convolution product, to the NavierStokes equations. The idea is to resolve larger-scale eddies and model (or filter) the smaller ones. The resulting filtered equations turn out to be functionally identical to the RANS/URANS equations, and an unknown subgrid scale stress $\tau_{i k}$ (and heat flux for compressible flows) must again be modeled. Like its RANS/URANS counterpart, for LES the most common model is the linear eddy viscosity type, which assumes the subgrid scale stress to be proportional to the local resolved rate of strain through a filter-dependent eddy viscosity. In most cases, then, it is solely in the modeling of the eddy viscosity that LES differs from RANS/URANS in a functional sense. This is the reason why it is so easy to program many types of blended RANS-LES models, because all that is usually required is a blending between the two different types of turbulence models. In LES, the turbulence models (usually referred to as subgrid scale models) typically include a built-in filter $\bar{\Delta}$, related to the local grid size (e.g., $\left.\bar{\Delta}=(\Delta x \Delta y \Delta z)^{(1 / 3)}\right)$. As 
$\bar{\Delta}$ decreases, the diffusion decreases and the model resolves smaller flow structures. Unfortunately, analysis of LES results is very easily complicated by numerics. Excessive numerical dissipation from the solver adversely affects the ability to resolve the desired large-scale features. In fact, numerical dissipation can itself behave like a subgrid scale model. This fact is taken advantage of in implicit LES (ILES) methods, which use no explicit subgrid scale models at all, and instead rely on the numerical scheme's inherent dissipation to provide the filtering needed at the smallest scales. Sometimes, as in the MILES approach, ${ }^{13}$ this reliance is theoretically justified, and sometimes is it just "assumed" that the inherent dissipation is appropriate. There can be very high demands in terms of grid spacing for LES. In particular, if the grid is too coarse to support the resolution of the dominant eddy structures, then the solution will be poor. One of the reasons why blended RANS-LES models have become very popular is that they significantly reduce the near-wall grid requirements by using RANS-like eddy viscosity in the near wall region rather than trying to resolve the (important, but very small) near-wall turbulent eddies. A fundamental challenge for blended RANS-LES models lies in defining the interface region between the two methods. It can be problematic, for example, if the interface region occurs within the log layer of a turbulent boundary layer. ${ }^{14}$ Much research is still ongoing in all of these areas today.

DNS is a direct numerical simulation of the Navier-Stokes (N-S) equations. At low Reynolds numbers, it is the same as solving for laminar viscous flow, but as the Reynolds number increases, turbulent eddies develop and are supported by the numerical scheme if the grid is fine enough and the numerical dissipation low enough. By implication and commonly-accepted standard working definition, DNS requires that the entire range of spatial and temporal scales of the turbulence must be resolved. The smallest scales are the Kolmogorov length and time scales: $\eta=\left(\nu^{3} / \varepsilon\right)^{(1 / 4)}$ and $\tau=(\nu / \varepsilon)^{(1 / 2)}$. However, resolving these scales means that the Reynolds numbers possible on today's computers are severely limited. Practical high Reynolds number computations using DNS will not be possible for many decades, by most estimates. Properly performed DNS also usually involves very high-accuracy numerical schemes because of the demands placed on resolving the small scales. Like in LES, dissipative numerics in DNS computations have very detrimental effects on a scheme's ability to resolve turbulent flow structures. This being said, it should be noted that it is becoming increasingly common to perform "under-resolved" or "coarse-grid" DNS. In this practice, the full Navier-Stokes equations are solved, but the grid (and possibly time step) is too coarse to resolve many of the smallest scales of motion. The argument in favor of this methodology is that the larger resolved scales have the majority of the influence on most of the relevant aspects of the flow field. It should be noted that there is a fine line between under-resolved DNS and ILES. The equations being solved in the code are often identical; neither method employs a turbulence (subgrid scale) model, and both methods are not resolving the finest scales, with the numerical dissipation presumably providing enough damping - but not too much - to prevent artificial build-up of energy at the smallest scales. In references, the distinction is sometimes drawn according to the numerical scheme employed. Often ILES is implemented in the framework of upwind schemes, through explicit artificial dissipation, or via low-pass frequency filters. ${ }^{15}$ On the other hand, "coarse-grid" DNS often utilizes high-order accurate low-dissipation schemes typical of DNS, but on grids too coarse to support the finest scales. But these distinctions are hazy, and it is possible, for example, to find an ILES computation with higher order and/or less dissipation than a "coarse-grid" DNS.

The purpose of this paper is to review the status of CFD as applied to flow control applications. Because this topic is so broad, this review will be accomplished by focusing solely on results related to CFDVAL2004. After the actual workshop in March 2004, many of the participants went on to refine their results for subsequent papers. Also, other workshops were held that included one of the cases. Finally, different CFD practitioners not associated with the original workshop have since computed one or more of the cases. Some of the questions this paper addresses are as follows. Has CFD gotten better at computing these types of flows in the last 4 years? Are more advanced methodologies being applied? What are the implications of these observations on the future of computations in this area? What challenges remain? An attempt was made to find and note all references involving CFDVAL2004 applications, although it is possible that some may have been inadvertently overlooked. Also, in cases where a conference paper was subsequently published in a journal, typically only the latter reference will be mentioned.

\section{Case 1: Synthetic Jet into Quiescent Air}

\section{A. General Description}

Case 1, 2-D synthetic jet into quiescent air from a thin slot with aspect ratio 28, at frequency $444.7 \mathrm{~Hz}$, was a difficult experiment to compute at the time of the workshop. The flow field was believed to be transitional, so it was unclear how best to simulate it. Workshop participants used N-S, LES, blended RANS-LES, URANS, and a reduced order model. As discussed below, end effects caused significant three-dimensionality away from where the jet emanated 
from the wall, but most participants computed the flow in 2-D. The side-mounted piezoelectric driver and complex plenum geometry, shown in fig. 2, were difficult to simulate, so all workshop computations made approximations inside the plenum or simply applied jet boundary conditions directly on the wall from which the jet emanated. As a result, the various computations often did not even start off with the same boundary conditions as the experiment at the jet exit: deviations from periodicity were for the most part not simulated. The many boundary conditions also varied significantly from each other. For URANS, it was therefore difficult to judge the capabilities of the turbulence models, which appeared to have a big impact on the results. See, for example, fig. 3, which shows ten different representative workshop results for time-averaged centerline vertical velocity. A very wide range of results was reported, some in better agreement with experiment than others (note that PIV and hotwire measurements differed substantially very near the slot). Computed turbulence quantities did not compare well at all with experiment.

It is important to note that additional measurements taken after the workshop, given in Yao, ${ }^{1}$ were at somewhat different conditions than those used for the workshop. Some of the larger discrepancies exhibited by PIV and LDV measurement techniques in the original data were mitigated in the later experiment, and it was determined that hotwire measurements were not as accurate very near the jet exit. The newer experiment produced a somewhat stronger jet with larger vertical velocities (by approximately $10-15 \%$ ). In subsequent CFD papers, some people compared with the original data and some compared with the newer data; both sets of data are available on the CFDVAL2004 website.

Plots showing the mean jet velocity contours from the newer experimental data are shown in figs. $4 \mathrm{a}$ and $\mathrm{b}$. As seen in the second figure, edge effects start to move toward the central part of the jet beyond a height of about $4 h$, where $h=1.27 \mathrm{~mm}$ is the slot width. The homogeneous central region does not appear to extend much above $8 h$ or so. This indicates that 2-D CFD (or configurations that ignore end effects) should not be expected to remain a reasonable approximation above this height. ${ }^{1}$ Three-dimensionality was suspected - but its extent was not known for sure - at the time of the original workshop.

\section{B. Low-Order and RANS Methods}

Yamaleev and Carpenter ${ }^{16}$ used a low-order method based on the 1-D Euler equations to compute the internal cavity flow, and combined it with the 2-D N-S equations (i.e., laminar) near and external to the slot. They used fourth-order upwind-biased finite-differencing based on Lax-Friedrichs flux splitting, and fourth-order Runge-Kutta time stepping. To use this low-order method successfully, they found that several conditions had to be met. These included the requirement that $R e_{d} \geq 500$ (where $d$ is the actuator orifice width), the interface between quasi-1-D region and 2D N-S region must be farther than $2 d$ below the orifice exit, and the actuator size should be much smaller than the diaphragm oscillation wavelength. Furthermore, they found that the cavity volume, neck length, slot size, diaphragm area, frequency, and amplitude all needed to be faithfully modeled. Their results were reasonable in the very-near field, but tended to degrade significantly further away from the slot exit.

Vatsa and Turkel ${ }^{17}$ used 2-D URANS to model the synthetic jet with two different turbulence models, SA and Menter's $k$ - $\omega$ shear stress transport (SST). ${ }^{18}$ Like most other people who computed this case, they did not faithfully model the 2-D section shape of the entire cavity. Vatsa and Turkel used the actual 2-D neck shape alone, and employed a periodic transpiration boundary condition at the bottom of the neck. At the workshop, their results were similar to many other participants, but they subsequently made an improvement to their unsteady boundary condition that brought their overall results in closer agreement with experimental data. The improvement was to adjust the periodic boundary condition at the bottom face of the cavity through the process of curve-fitting the measured velocity at the slot exit (using the newer experimental data) with a fast Fourier transform (FFT). In this way, the proper mode shapes from the experiment were better modeled. They also enforced zero net mass flow. In the original workshop, most participants used a simple sine wave transpiration boundary condition, Even when allowing for large experimental measurement uncertainties, results from these earlier computations did not appear to match the experimental data at the slot exit very well. The actual drum-like diaphragm, combined with the complex three-dimensional cavity shape, likely produced additional modes that affected the flow field emanating from the slot. By using this improved boundary condition, Vatsa and Turkel were able to achieve better agreement with experimental data, as shown in fig. 5 for time-averaged vertical velocity along the centerline. SA results are shown in this figure. SST results were nearly the same in the near-field (out to approximately $y=10 \mathrm{~mm}$ ), but were slightly worse than SA beyond that. However, the flow field's three-dimensionality beyond $y=10 \mathrm{~mm}$ would require 3-D computations to model the end effects correctly in any case. Although not shown, results also agreed well with phase-averaged quantities in the two-dimensional near-field. In a separate paper, Vatsa and Carpenter ${ }^{19}$ also looked at the effect of higher order temporal schemes, but found very little effect.

Zhang and Wang ${ }^{20}$ used 2-D URANS with a $k$ - $\omega$ turbulence model, solved via second-order upwind discretization. Their cavity geometry was very different from the actual configuration, but they employed the same FFT technique 
used by Vatsa and Turkel to determine the appropriate boundary condition. As a result, they matched phase-averaged velocity profiles and time-averaged vertical velocity along the centerline very well (compared to LDV experimental data) in the region near the wall. Their validation against case 1 was only a part of their paper; the main focus was to explore novel signal wave patterns for synthetic jets.

Park et al. ${ }^{21}$ used 2-D URANS with several different turbulence models, including SA, SST, and a linear version of a $k-\varepsilon$ model. They used cell-centered finite volume with third-order MUSCL HLLE plus preconditioning, and second order dual time stepping. Their cavity geometry and grids were very similar to those used by Vatsa and Turkel. However, their boundary condition was not obtained through the use of an FFT. Instead, they attempted to create a simple function that would produce a close curve fit to the data at the exit:

$$
v(t)=\frac{A}{\Theta^{0.55}} \sin (2 \pi f t)
$$

where $\Theta=\max (0.25, \theta /(2 \pi))$, and $\theta$ is the phase. Although this method did not necessarily include modal behavior, it likely contributed to producing better agreement than a simple sine function would have done. With the exception of the $k-\varepsilon$ model, their results were very reasonable. The biggest effects due to turbulence modeling were in the region away from the wall where 3-D end effects are significant.

Carpy and Manceau ${ }^{22}$ investigated case 1 using 2-D URANS with a $k$ - $\varepsilon$ model and a full Reynolds stress moment (RSM) closure model. They did not solve for flow in the cavity at all, but rather applied a Dirichlet boundary condition directly at the slot exit. This boundary condition was based on the (original) experimental data measured near the slot exit. Using the experimental data for the boundary conditions was necessary because the fluid was mainly blown out from the center of the slot and sucked in from the sides, yielding complicated non-sinusoidal behavior. They also used $u_{i}^{\prime} u_{j}^{\prime}$ from experiment, reconstructed the missing $w^{\prime 2}$ data by assuming $w^{\prime 2}=u^{\prime 2}$, and obtained $\varepsilon$ boundary conditions from $k^{3 / 2} / L$, with $L$ half of the slot width. They solved the incompressible form of the equations, using second-order central differencing for convection and either second or first order time stepping. They noted that $k-\varepsilon$ predicted a toorapid decrease in the peak velocity according to phase-averaged velocity profiles, and thus too short a penetration of the jet into quiescent air. It also underpredicted the jet convection velocity above the slot. The RSM performed much better, particularly with regard to predicting acceleration above the slot (although somewhat underestimated compared to experiment). Fig. 6 shows vertical velocities at eight different phases during the cycle. Here, the phases are defined such that peak blowing velocity at the slot exit occurs near phase $=45$ deg. Although both models underpredicted peak velocity and convection velocity, the misprediction was particularly severe using $k-\varepsilon$. They believed the flow to be more transitional than fully turbulent. They performed analysis of momentum budget and also looked at the misalignment of the strain and anisotropy tensors. One of their main conclusions was that in flows like this - subject to periodic irrotational strains - a time lag exists between the strain and anisotropy tensors. Negative production results from this misalignment, and $k-\varepsilon$ as well as other linear turbulence models cannot account for this behavior. They also noted that the turbulence is far from equilibrium in this flow, so even the RSM has some difficulty accurately modeling the interaction between turbulence and the vortex dipole.

\section{Blended RANS-LES and LES Methods}

Xia and Qin ${ }^{23}$ solved 3-D detached eddy simulation (DES) ${ }^{24}$ using an unstructured second-order finite volume scheme with flux difference splitting and dual time stepping with second-order Runge-Kutta. In DES, the solution equations vary automatically between SA near walls and a Smagorinsky-type of LES model away from walls, according to a modified length scale in the original SA model: $\tilde{d}=\min \left(d, C_{D E S} \Delta\right)$, where $\Delta$ is related to the local grid spacing. Xia and Qin used a two-dimensional version of the actual cavity shape, with the vibrating membrane on the side, like in the experiment. They employed a moving diaphragm boundary condition, with realistic drum-like motion in two dimensions: $x=A F(t) \cos \left[\pi\left(y-y_{0}\right) / L\right]$. They obtained the maximum oscillation amplitude $A$ from the experimental measurement of the diaphragm center. This represents the only computation known for which a priori knowledge of the jet exit flow field was not used in helping to determine the plenum boundary condition; in other words, their boundary condition was truly predictive. However, the actual shape was not modeled accurately in the third dimension; instead, the two-dimensional shape was repeated laterally and periodic boundary conditions were applied on each of the three-dimensional sides. They also ran 2-D laminar flow N-S for comparison, but these results were not as good as DES and are not discussed here. Even in 3-D, the flow inside the cavity and neck was very two dimensional. Due to the relatively low Reynolds number and the favorable pressure gradient in the outgoing flow, it was difficult for transition to occur prior to exiting the slot. Use of DES resulted in LES-like three-dimensional structures quickly forming after the flow emerged from the slot. Time-averaged results agreed well with the (original) experimental PIV data up to $y=8 \mathrm{~mm}$, beyond which PIV data was not taken. They did not perform quantitative 
comparisons with data beyond this location. However, because they used periodic boundary conditions, they were not simulating end effects. They claimed that the primary mechanism for turbulent eddy generation is the instability and breakdown of the vortex pairs that occurs during the suction phase.

Cui and Agarwal ${ }^{25}$ performed 3-D DES and blended SST-LES ${ }^{26}$ simulations for this case, along with several 2-D and 3-D URANS runs. They used a second-order approximate-factorization scheme, with Euler implicit time stepping. Like Xia and Qin, for their 2-D runs they used a two-dimensional version of the actual cavity shape, with the boundary condition on the side wall. However, Cui and Agarwal did not use a moving wall boundary condition, but rather employed a sinusoidal transpiration boundary condition and tuned the pressure at the diaphragm face in order to achieve zero net mass flux over one cycle. They adjusted the amplitude of the velocity boundary condition in an attempt to match the experimental data at the slot exit as closely as possible, but, as was discovered by the many participants at the CFDVAL2004 workshop, this proved to be very difficult to do. They ended up with a result that partially matched the original PIV data and partially matched the original hotwire data. As discussed earlier, subsequent experiments ${ }^{1}$ were able to achieve good agreement between PIV and LDV measurements, but hotwire measurements were believed to be less reliable near the jet exit. So the fact that hotwire was partially used to determine the boundary condition here may have caused some discrepancies. Results using different turbulence models and the two blended RANS-LES methods varied significantly, and none matched the data as well as others whose boundary conditions were tuned to agree better with the experiment at the jet exit. Regarding the 3-D computations, Cui and Agarwal were the only researchers to attempt to model the entire 3-D problem. They modeled the actual cavity geometry (albeit employing a simple transpiration boundary condition), and included the slot ends without use of periodic boundary conditions. However, their grids were fairly coarse in this regard - only 29 grid points along the length of the slot.

\section{Navier-Stokes Simulations}

Kotapati et al. ${ }^{27}$ performed 3-D N-S simulations using an incompressible cell-centered, collocated solver that used a weighted averaging of second-order central differencing and second-order upwind differencing. The method was second order in time. They used a different cavity and neck shape than the experiment that were only nominally representative of the actual geometry, with simple sinusoidal transpiration boundary conditions specified at the bottom of the cavity. They attempted to achieve a close match with the original PIV and LDV data near the slot exit, with the better agreement generally with the PIV data. The cavity shape was repeated in the third dimension, and periodic boundary conditions were employed in that direction. They looked at spectra from their computations near the slot, and found that the simulations resolved scales deep into the dissipation range. In other words, in the near field close to the jet exit plane, they argued that the simulation effectively constituted a DNS. Their finest grid had a little over 1.5 million cells, and they investigated the effects of a variety of numerical parameters. They used a very small time step corresponding to 14000 steps per period, and included a small sinusoidal perturbation in the beginning of each run to induce the three-dimensionality. Approximately 10 cycles were run to eliminate transient behavior, and 6 cycles were used to accrue statistics. This amount appeared to be sufficient to converge the statistics in the near-field. One of their conclusions was that matching key nondimensional parameters - particularly Strouhal number - appears to be sufficient for capturing critical features of the external jet flow, at least in the incompressible regime. They also found that the counter-rotating vortex pairs break down and transition to turbulence due to spanwise instabilities. They only compared with experiment below $y / h=6$ or so, because they did not model the end effects, and comparisons were generally very good. They found the turbulence was not well-developed within the slot, but according to the spectra the flow breaks down rapidly into a well-developed jet a short distance from the slot exit. They did not compare with experimental turbulence data taken in the flow field. A figure showing vortical structures in the slot exit region from their computation is given in fig. 7. Isosurfaces of the imaginary part of the complex eigenvalue of the instantaneous velocity gradient tensor are plotted. Regarding nondimensional parameters, they found the Strouhal number to be the most important for determining vortex celerity (i.e., speed); the Reynolds number alone had little effect.

\section{Case 2: Synthetic Jet in a Crossflow}

\section{A. General Description}

Case 2, 3-D circular synthetic jet with frequency $150 \mathrm{~Hz}$ into turbulent crossflow boundary layer, was the leastcomputed of the three workshop cases, probably because it was necessarily three-dimensional. Most workshop participants used RANS, and one used LES. At the center of the jet orifice exit, the experiment exhibited a large cross-flow velocity (with peak the same order of magnitude as $u_{\infty}$ ) of unknown origin, which was not modeled in any of the CFD simulations. The influence of this cross-flow component on the flow field solution is not known. Qualitative agreement 
of CFD with experiment was reasonably good, but quantitative comparisons showed significant variations. Different turbulence models were found to have less of an impact than different grids, codes, or other solution variants. LES and RANS solutions on similar-sized grids yielded very similar results in mean-flow quantities. There have only been a handful of papers published on case 2 since the time of CFDVAL2004.

A sketch showing the case 2 arrangement is given in fig. 8 . Inside the plenum, the floor, made up of a solid square plate mounted on a flexible membrane, was driven electromechanically from below. This motion caused a considerable change in cavity volume, by more than a factor of two (the cavity was approximately $1.7 \mathrm{~mm}$ deep, and the floor moved approximately $\pm 0.77 \mathrm{~mm}$ ).

\section{B. RANS Methods}

Iaccarino et al. ${ }^{28}$ used RANS modeling for both case 2 and case 3 in two different second-order codes using several different turbulence models. For case 2, both a structured and an unstructured grid were employed. In the structured grid, no plenum was computed and uniform sinusoidal normal velocity was specified at the plate surface. In the unstructured grid, a plenum was computed with sinusoidal mass flow imposed at the floor. They did not show many results, but - much like results at the CFDVAL2004 workshop - time-averaged streamwise velocity profiles compared only in a qualitatively sense with experiment. The V2F turbulence model ${ }^{29}$ appeared to perform somewhat better than a $k-\varepsilon$ model, but different codes were used so it is impossible to draw a firm conclusion.

Biedron et al. ${ }^{30}$ employed a second-order unstructured code using flux difference splitting and the SA turbulence model. They used dual time stepping and temporal error control as an exit criteria for determining the number of subiterations required to drive algebraic errors below a specified fraction of the temporal error norm. They used periodic velocity transpiration boundary conditions prescribed on the floor of the plenum in an attempt to match the peak vertical velocity out of the center of the orifice as measured in the experiment. Like Iaccarino et al. and the earlier workshop participants, their limited results showed qualitative agreement with experimental data. They also noted large differences due to grid size for some quantities; their fine grid had over 255000 nodes whereas their coarse grid had 46000 nodes.

Rumsey ${ }^{31}$ employed a second-order structured code using flux difference splitting and three turbulence models, including SA, SST, and an explicit algebraic stress model (EASM) based on $k-\omega \cdot{ }^{32}$ Dual time stepping was employed in conjunction with multigrid. A steady-RANS-like turbulent profile was specified at inflow. The computations modeled the cavity, and a sinusoidal transpiration boundary condition was applied on its floor. As a result of this boundary condition, the computations matched the minimum and maximum vertical velocities at the orifice exit, but other aspects of the experimental data were missed (including the large unexplained cross-flow $v$-velocity component). Comparisons with the $u$ (streamwise) and $w$ (vertical) velocity components above the orifice exit are shown in fig. 9. Here, two grid sizes were used: the fine grid had 4.1 million points and the medium grid had 530,000 points. Peak blowing velocity at the slot exit occurs near phase $=115 \mathrm{deg}$. in the experiment. With these levels of agreement at the jet exit (similar to the agreement shown by others at CFDVAL2004), how well do we expect to predict the rest of the flow field? A large number of results throughout the flow field were shown, and the effects of grid, time step, and number of subiterations were also explored. In summary, many global flow features were successfully captured, in terms of time-averaged, phase-averaged, and time-dependent quantities, but there were many quantitative differences. Examples are shown in fig. 10, where the general trends are seen to agree reasonably well, but, for example, peak $w$-velocity as a function of phase is underpredicted. Results on the finer grid (f) were better than those on the medium (m) grid. Although the three turbulence models investigated produced some differences, these differences were generally not too large, so it was not possible to isolate one turbulence model as being better for this flow. URANS was particularly unsuccessful at predicting turbulence quantities. A later publication by Rumsey et al. ${ }^{33}$ contrasted case 3 with a similar synthetic jet experiment conducted at NASA Glenn Research Center. It was emphasized that there were differences between case 2 PIV and LDV measurements in some regions of the flow, and the URANS computations tended to agree better with the LDV data in those regions. Including the internal orifice in the computations was shown to be important for capturing the complex nature of the flow field in its immediate vicinity, particularly during expulsion.

\section{Blended RANS-LES and LES Methods}

Cui and Agarwal ${ }^{34}$ employed a second-order structured code in conjunction with both URANS (SST) and blended RANS-LES (DES). They computed the cavity and used a sinusoidal transpiration boundary condition on its floor. They also tuned the pressure at this boundary to insure a zero net mass flow during one cycle. DES was activated zonally; i.e., it was only active in the zones inside and immediately outside the orifice, and standard SA was used everywhere else. They did not show many results, but both the URANS with SST and blended RANS-LES with DES 
appeared to yield generally very similar results in terms of $u$ and $w$ velocity components. Although neither model accounted for the large unexplained cross-flow $v$-velocity component from experiment at the orifice exit, downstream the DES yielded results in the $v$-velocity component that were much closer to experiment than the URANS.

Similar to their work for case 1, Xia and Qin ${ }^{35}$ used DES to compute case 2 using an upwind scheme with gradientbased linear reconstruction and dual time stepping. They employed a moving diaphragm boundary condition, with sinusoidal motion as prescribed in the experiment. This boundary condition gave reasonable representation of the vertical velocity component measured above the center of the orifice, very similar to others who used transpiration boundary conditions; it did not mimic the double-hump feature seen in the experiment (see fig. 9b). Very few quantitative results were shown.

Dandois et al. ${ }^{36}$ performed both URANS (with SA) and LES (with a selective mixed-scale subgrid scale model) in a second-order code based on the AUSM+(P) scheme. The code was second order temporally accurate with Gear's implicit scheme solved by an approximate Newton method. At the turbulent inflow boundary, a steady-RANS-like turbulent profile was specified for most of the computations (including LES), although one LES simulation seeded unsteady turbulent fluctuations there as well. In the former case, the turbulent boundary layer leading up to the orifice contained no turbulent structures; in the latter case it did. The cavity was computed with a sinusoidal transpiration boundary condition on its floor. When comparing LES and URANS in the flow field, both were found to capture the main structure of the counter-rotating vortex pair, with URANS giving less detailed structure as expected. Both methods were similar in terms of mean profiles, but the seeded LES was significantly better in terms of predicting the turbulence statistics. Examples are shown in figs. 11 and 12. In these figures, LES-M1+F indicates LES on fine grid with seeding at inflow, LES-M1 indicates LES on fine grid, LES-M2 indicates LES on medium grid, LES-M3 indicates LES on coarse grid, and URANS-M3 indicates URANS on coarse grid. Grid resolution appeared to be the dominant parameter for adequately predicting the transport of the vortical structures. They hypothesized that the unsteadiness of the velocity field is driven primarily by the synthetic jet, and not by turbulence fluctuations, which is why URANS appears to be sufficient to simulate the mean flow dynamics. In their paper the authors also attempted to make a correction to the experimental data, to determine the effect of the large unexplained cross-flow $v$-velocity component from experiment at the orifice exit. They assumed a nonvertical piston displacement and performed an axis rotation of the coordinate system in order to force $v=0$. By doing this, they found that the severe "dip" in the $w$-velocity profiles near phase $150^{\circ}$ (see fig. 9b) was lessened.

\section{Case 3: Flow over a Hump Model}

\section{A. General Description}

Case 3 was flow over a nominally two-dimensional wall-mounted hump, inspired by earlier similar experiments of Seifert and Pack. ${ }^{37}$ The Reynolds number was somewhat less than 1 million based on chord. On this model, the flow (with no control) separates near 65\% chord, and reattaches downstream past the end of the hump. See sketch in fig. 13. The baseline condition of no flow control was one of the tests during the CFDVAL2004 workshop. Also, flow control applied near the separation point can lessen the size of the separation bubble. For CFDVAL2004, one particular suction case and one particular oscillatory control case were used, but other variations were tested in the experiment. After the CFDVAL2004 workshop, the experimental data from case 3 were included as part of the ERCOFTAC on-line database (Classic Collection) ${ }^{\mathrm{b}}$, and the data were also included as test cases in two subsequent workshops: the 11th ERCOFTAC/IAHR Workshop on Refined Turbulence Modelling in Goteborg, Sweden, 2005, ${ }^{38}$ and the 12th ERCOFTAC/IAHR Workshop on Refined Turbulence Modelling in Berlin, Germany, 2006. ${ }^{39}$ It was noted in the experiment that end plates used to improve flow two-dimensionality also caused blockage effects, particularly noticeable in surface pressure levels over the hump. In computations, some people accounted for this blockage (for example, by contouring the upper wall shape in 2-D computations), and some people did not. In any case, experiments with and without end plates indicated little effect on separation and reattachment locations. ${ }^{3}$

The hump case has been computed by no less than 16 different groups. During the CFDVAL2004 workshop the case demonstrated a failing of RANS/URANS turbulence models in general: the eddy viscosity in the separated shear layer region was significantly underpredicted in magnitude, leading to too little mixing and hence too late a reattachment downstream. An example of the shear stress magnitude underprediction by almost all workshop participants using the SA model was shown earlier in fig. 1. This same significant failing has also been demonstrated for another similar 2-D flow with separation (but no flow control). ${ }^{40,41}$ At the CFDVAL2004 workshop, blended RANS-LES and under-resolved DNS computations appeared to offer some hope for improved capability in this regard, but at the time

\footnotetext{
${ }^{b}$ http://cfd.mace.manchester.ac.uk/ercoftac/ [cited 6/2008].
} 
these methods were not shown definitively to be superior to RANS/URANS, since reattachment lengths were still for the most part too long. As will be shown below, however, improvements have subsequently been made in these areas.

\section{B. RANS Methods}

All of the results described in this section were computed in 2-D. As mentioned earlier, Iaccarino et al. ${ }^{28}$ used RANS/URANS to compute both cases 2 and 3. They primarily computed the previous hump experiments of Seifert and Pack, ${ }^{37}$ which were very similar to case 3 but at generally higher Reynolds and Mach numbers (and also having a few minor geometrical differences near the slot). ${ }^{3}$ They computed both with and without the plenum included; when not included, the boundary conditions were specified for constant suction through velocity and density conditions, assuming zero pressure gradient. Very limited results for CFDVAL2004 case 3 showed the SA model to be in better agreement with experimental surface pressure data than a $k-\omega$ model, but both were deficient in the separated region.

Capizzano et al. ${ }^{42}$ used the same second-order structured-grid code with dual time stepping employed by Iaccarino et al.. They did not model the plenum. Their main contribution was the development and application of various Neumann surface boundary conditions for steady suction and oscillatory control for which jet angle and other aspects like spatial distribution could be controlled. The SST model was employed for all runs, and results were consistent with other workshop RANS/URANS computations.

Cui and Agarwal ${ }^{43}$ employed a second-order structured code in conjunction with SST for the no-control and oscillatory-control conditions. Surface pressures were severely overpredicted, possibly due to a combination of setting incorrect back pressure boundary conditions and not accounting for blockage. However, limited velocity profiles appeared to be consistent with other workshop RANS/URANS computations, in the sense that computed results remained separated too long.

Balakumar ${ }^{44}$ was one of only two workshop participants to compute case 3 using a higher order spatial scheme. He employed fifth-order WENO, with third-order TVD Runge-Kutta for time integration. Surface boundary conditions were applied rather than computing a plenum; a $\sin ^{2}$ velocity distribution was employed normal to the surface. Results with the SST model yielded very similar results to other workshop RANS/URANS computations. Reattachment was delayed, and the magnitude of the turbulent shear stress was dramatically underpredicted in the separated region.

Morgan et al. ${ }^{45}$ also applied a higher-order scheme, using compact finite differences. They explored the differences between using second and fourth order discretization, both for the mean flow as well as for the $k-\varepsilon$ turbulence model (uncoupled from the mean flow). They included the plenum in their computations. They demonstrated that a second-order discretization of the turbulence model dominated the solution even when higher order differencing was used for the mean flow equations. Their results in terms of reattachment were consistent relative to other workshop RANS/URANS computations.

Bettini and Cravero ${ }^{46}$ used commercial software in conjunction with structured grids and several turbulence models. Details about the numerical method were not given. The plenum was included in the computations. Results using a RNG $k-\varepsilon$ model for the baseline condition were very poor compared to experimental data, with severe underprediction of the surface pressures over the attached portion of the hump and a too-rapid rise in pressures in the separated region. Steady suction results were better, but still tended to have somewhat large pressure overshoots in the separated region. They also looked at different suction levels from the experiment. For oscillatory control, they explored the effects of $k-\varepsilon$, SST, and an RSM. The RSM was found to produce a smaller time-averaged bubble length than SST.

He et al. ${ }^{47}$ used the same commercial software; it was used as a segregated solver, with second order upwind and a SIMPLE algorithm for pressure-velocity coupling. They only computed the baseline condition from CFDVAL2004, then performed different computations with plasma flow control to model a different experiment conducted by them on this same hump shape. They used the $k-\varepsilon$, SA, and SST models and did not include a plenum. Results for the baseline condition were very reasonable, which brings into question why the same commercial software yielded different results for Bettini and Cravero. One possible cause may have been the use of different turbulence models. SA and SST reattached too late, in agreement with other workshop RANS/URANS computations. In this case $k-\varepsilon$ agreed well with the experimental reattachment location. However, it appears that it separated late. In other words, $k-\varepsilon$ likely got the right result (early reattachment) for the wrong reason (late separation). A similar trend was uncovered for a 2-D hill case at a different workshop. ${ }^{40}$ In any case, the turbulent shear stress predictions for $k-\varepsilon$ in the separated region were no better than those of SA or SST; all underpredicted the magnitude seen in the experiment.

Madugundi et al. ${ }^{48}$ also used the same commercial software. They did not compute a plenum. For the steady suction case they applied a surface velocity boundary condition angled $30^{\circ}$ to the surface facing downstream. They investigated several different turbulence models for both the baseline and suction conditions. They also computed different cases unrelated to CFDVAL2004. Their case 3 results saw significant variations for the different turbulence models. In general, they obtained the surprising result for the baseline case that most of their computations appeared 
to reattach early compared with experiment (looking at velocity profiles). This trend is inconsistent with all the other previous workshop RANS/URANS computations, as well as with the He et al. results using the same commercial software.

Rumsey ${ }^{49}$ focused primarily on the oscillatory control condition from the workshop, then subsequently Rumsey and Greenblat $t^{50}$ looked at many of the other steady suction and oscillatory control conditions from the experiment not included in CFDVAL2004. This latter study was conducted in order to determine if RANS/URANS, although not accurate in predicting reattachment, could predict trends due to variations in flow control parameters. A second-order structured-grid code with flux difference splitting and dual time stepping was used, and the plenum was included in all computations. Three different turbulence models were employed: SA, SST, and EASM based on $k-\omega$. Results upheld the earlier workshop conclusions that all models produced insufficient eddy viscosity in the separated region and reattachment occurred too late. Like most others who had computed the oscillatory control condition, the general mean-flow time-dependent character appeared to be captured reasonably well in spite of the overprediction of reattachment length. A numerical experiment demonstrated that SA with eddy viscosity arbitrarily doubled in the separated region yielded earlier reattachment, in better agreement with experiment. This test seemed to confirm that it is at least possible that a RANS/URANS turbulence model could be devised to give better agreement for these types of cases. Regarding trends, for steady suction CFD appeared capable of predicting variations due to Reynolds number and suction strength $C_{\mu}=\rho_{j} h U_{j}^{2} /(c q)$, where $U_{j}$ is the total jet (suction) velocity at the slot. Examples showing the trends as a function of $C_{\mu}$ are shown in figs. 14 and 15. As $C_{\mu}$ increased, the bubble size decreased in both experiment and computations. The only experimental data available were at the lowest $R e$ of 557,400 and 936,000. The experimental results appeared roughly linear on this $\log$ plot. The CFD results were also fairly linear at the lower $C_{\mu}$, but they tended to drop down at the higher $C_{\mu}$. Overall, CFD produced a slightly shallower slope than experiment, along with a significantly longer bubble at the same $R e$. The change between $R e$ of 557,400 and 936,000 was similar for CFD as experiment. Coincidentally, the $X_{B}$ levels from CFD at the highest $R e$ matched fairly well with the experimental levels at the lowest $R e$. URANS results for oscillatory control trends were not as favorable.

\section{Blended RANS-LES and LES Methods}

Since the time of the CFDVAL2004 workshop, many papers have come out using blended RANS-LES or LES methods for case 3. Israel et al. ${ }^{51}$ used both a "coarse-grid" DNS (later re-done and published separately, as discussed in the next section) and a blended RANS-LES method referred to as flow simulation methodology (FSM). In FSM the stress tensor from the underlying RANS model (in this case EASM based on $k-\varepsilon$ ) was multiplied by a contribution function

$$
f_{\Delta}=\frac{\beta \bar{\Delta}^{2 / 3}-\eta^{2 / 3}}{L^{2 / 3}-\eta^{2 / 3}}
$$

where $\beta$ is a constant and $L$ is the turbulence length scale. They used a second order code based on flux difference splitting and dual time stepping. Boundary conditions were applied directly on the wall, and a RANS profile was specified at the upstream boundary. Periodic boundary conditions were specified at the sides of the domain, which was $0.17 c$ wide. FSM was successful at developing eddy content downstream of separation. However, time-averaged results were not improved relative to RANS/URANS results; reattachment still occurred too far downstream. The reference discussed issues associated with error estimation and analysis for a stochastic problem such as this.

Hiller and Seitz ${ }^{52}$ employed a scale adaptive simulation (SAS) ${ }^{53}$ blended RANS-LES model in an unspecified unstructured-grid code. They ran the oscillatory control case on a domain one-third of the channel width, or about $0.56 c$, with periodic bounday conditions. SAS depicted the development of streak-like structures of the turbulent flow, and appeared to reduce the bubble length compared to URANS with SST, although SAS results were still somewhat too long. Only very limited quantitative comparisons were shown. They also investigated the effects of higher frequency actuation and actuation with a duty cycle.

Biswas $^{54}$ used an incompressible fifth-order WENO code with upwind-biased finite differencing (fourth-order central differencing on viscous terms) to solve the LES equations for case 3. The subgrid scale model was based on an additional equation for turbulent kinetic energy, and the coefficient was evaluated through a dynamic procedure. The plenum was included, and periodic boundary conditions were applied to the spanwise boundaries located $0.2 c$ apart. Results were spectacularly good. In fact, computed results essentially matched all experimental results (slightly offset) even for the jumps and wiggles due to instrument bias and/or imperfections in the as-built model. Because the CFD could not possibly have accounted for all these experimental variations, judgement is suspended on this work until further information or confirmation is obtained. 
Krishnan et al. ${ }^{55}$ performed a large number of computations for the CFDVAL2004 workshop, including both RANS/URANS and DES. For RANS/URANS, they ran many variations, including structured, unstructured, 2-D, 3D periodic spanwise, and even 3-D with end plates included. It is in large part due to their complete collection of submitted cases that the important effect of blockage due to the end plates was discovered. Their 3-D results with end plates not only improved surface pressure comparisons over the front part of the hump, they also led to good comparisons in the separated region as well. Others who accounted for end plate blockage solely through using end plate cross section area to modify top wall shape in 2-D computations saw good agreement over the front part but not in the separated region. Further details on the RANS/URANS results of Krishnan et al. are not discussed here, other than to say that they were completely consistent with the majority of other RANS/URANS results. Their method employed a second-order compressible code, with an exact Riemann solver and second order time accuracy with Newton subiterations. They included the plenum in the computations, but noted that for relatively small values of mass flux a prescribed wall boundary condition would be a valid alternative. However, they felt that for higher mass flux, impedance effects become significant and a plenum should be included. For DES they ran with a $0.121 c$ spanwise extent and periodic boundary conditions on the side planes. They needed to turn off DES upstream of the slot to get it to work for this case. This was because otherwise, on their particular grid, the RANS-LES interface was too low in the boundary layer at the upstream locations, corrupting the solution there. Results for the baseline case were good in comparison with experiment: velocity profiles, turbulent shear stress profiles, and bubble length were all significantly improved compared to RANS/URANS. However, steady suction results were not much improved. Smaller separations like the one in the suction case appear to be problematic for DES. A future fix may be to seed eddy content into appropriate regions of the upstream flow so that there may be sufficient eddy content by the time it gets to the small separated region. DES was not employed for the oscillatory condition.

Saric et al. ${ }^{56}$ solved incompressible RANS, DES, and LES using a cell centered collocated finite volume scheme with a SIMPLE algorithm for coupling the velocity and pressure fields. For LES, they use the Smagorinsky subgrid scale model with constant coefficient $C_{s}=0.1$. Convection transport was solved in most cases with second order central differencing, and Crank-Nicolson was used for temporal differencing. Flow control boundary conditions were applied at the surface. For DES and LES they applied periodic boundary conditions in the spanwise direction; for DES the width was $0.2 c$ and for LES it was $0.152 c$. The latter was subsequently found to be insufficient to ensure spanwise decorrelation. For DES and LES the upstream boundary condition used steady profiles from experiment. It is not clear how/where transition was induced in the LES computations. Statistics were gathered over $5-7$ flow-through times. For LES their grid spacing near the wall yielded: $\Delta x^{+}=80, \Delta y^{+}=1$, and $\Delta z^{+}=50$. The first and last of these were somewhat high compared to recommended levels for capturing streaky structures near walls. In spite of the coarseness of the grids used (which may have caused too high stress levels prior to separation), LES significantly outperformed the RANS/URANS models tested, which behaved like other previous workshop RANS/URANS computations. In particular, LES predicted higher turbulent shear stress magnitudes in the separated region, in good agreement with experiment, and yielded shorter bubble lengths. DES performed similarly well for the baseline case, but, like in Krishnan et al., not for the steady suction case. This latter failure highlighted the importance of grid design when using DES: in this case the suction thinned the boundary layer and separation region, so the RANS-LES interface in DES ended up too far from the wall.

Morgan et al. ${ }^{57}$ performed compressible ILES computations with a fourth-order compact differencing scheme. This method relies on a high-order nondispersive low-pass Pade-type spatial filter in lieu of a subgrid scale model to prevent build-up of energy at the unresolved high wave numbers. The spatial filter is of the form

$$
\alpha_{f} \bar{Q}_{i-1}+\bar{Q}_{i}+\alpha_{f} \bar{Q}_{i}+1=\sum_{n=0}^{N} \frac{a_{n}}{2}\left(Q_{i+n}+Q_{i-n}\right)
$$

where $N$ provides a $2 N$ th-order filter, and $-0.5<\alpha_{f}<0.5$. The higher $\alpha_{f}$, the less dissipative the filter. They used $\alpha_{f}=0.4$, in a 6th-order filter. Time stepping was done with second-order implicit Beam-Warming approximate factorization with three Newton-like subiterations. In order to resolve the flow on a reasonable-size grid with LES, they found it necessary to run at about one-fifth the Reynolds number in the experiment. They used periodic boundary conditions in the spanwise direction, with span width $1.39 \mathrm{c}$. The grid spacing near the wall yielded: $\Delta x^{+}=60$, $\Delta y^{+}=1$, and $\Delta z^{+}=20$. They included the plenum in the computations, and at the inflow boundary they used a steady state RANS solution profile with flow disturbances from a flat plate LES superimposed. Baseline and steady suction results were very good in comparison with experiment, but the oscillatory control case was predicted with too much separation (although LES results were better than RANS/URANS). Inside the separation bubbles, LES predicted turbulent shear stress and turbulent kinetic energy profiles significantly better than RANS/URANS. Similar to Saric et al., upstream of the slot the LES dramatically overpredicted turbulent shear stress levels compared with experiment. 
They hypothesized this to be due to possible unsteady motion of the separation point, which at the lower Reynolds number is located farther upstream than at the Reynolds number in the experiment. Surface skin friction levels in the front curved region of the hump were overpredicted, which they also attributed to running at too low a Reynolds number.

You et al. ${ }^{58}$ performed incompressible LES with an energy-conservative second-order central difference scheme on a staggered mesh. Aliasing errors were controlled by enforcing kinetic energy conservation as opposed to numerical dissipation or filtering, thus avoiding artificial damping of small scales. Temporal differencing was a fully implicit fractional step Crank-Nicolson method. The subgrid scale model was a dynamic Smagorinsky model. They did not include the plenum in the computations, and periodic boundary conditions were applied in the spanwise direction. Spanwise extent was $0.2 c$. At the inflow, a recycling procedure was employed. The grid spacing near the wall yielded: $\Delta x^{+} \leq 50, \Delta y^{+} \leq 0.8$, and $\Delta z^{+} \leq 25$. They noted that a non-uniform dynamic Smagorinsky coefficient resulted throughout the flow field, so using a constant coefficient model is probably not a good approximation. Overall, results were excellent, perhaps with the exception that surface skin friction was overpredicted near the front of the baseline hump (like in Morgan et al.). Surface pressures, mean and turbulent profiles, and bubble length were all predicted in very good agreement with experiment. You et al. also plotted various quantities in comparison with Krishnan et al., Saric et al., Morgan et al., and Capizzano et al.. A few of these are repeated here, in figs. 16 and 17. The You et al. bubble size and shape for steady suction is shown in fig. 18 along with experimental results. Agreement is very good.

Franck and Colonius ${ }^{59}$ performed compressible LES simulations with a non-density-weighted, low-pass-filtered method with a sixth-order Pade scheme in the wall-normal direction, fourth-order explicit treatment in the streamwise direction, and a Fourier spectral method in the spanwise direction. They employed the same type of spatial filter used by Morgan et al., except they used eighth order with $\alpha_{f}=0.45$. Time stepping was fourth order Runge-Kutta. Either a Smagorinsky subgrid scale model with fixed or dynamic coefficients was employed (averaged over the spanwise direction), or else no model (ILES) was used. The spanwise extent of the domain was $0.2 c$, with periodic boundary conditions. Jet boundary conditions were specified directly at the wall. Upstream, they did not fully resolve the turbulent boundary layer; instead, they initialized the velocity profile at the wall with velocity perturbations formulated from random Fourier modes, which accelerated development of turbulence. They found little difference between results using LES with or without a subgrid scale model. Agreement with surface pressure coefficients and bubble length for the baseline and steady suction conditions were very good. Similar to Morgan et al., for oscillatory control results were not as good, overpredicting reattachment location and giving spanwise vorticity consistently higher than experiment. They also explored issues related to compressibility by running higher Mach numbers and comparing with Seifert and Pack ${ }^{37}$ data.

\section{Navier-Stokes Simulations}

As a follow-on to work ${ }^{51}$ they performed for the CFDVAL2004 workshop, Postl and Fasel ${ }^{60}$ used over 200 million grid points in a "coarse-grid" DNS simulation of case 3. In other words, they performed a direct simulation of the incompressible vorticity-velocity form of the Navier-Stokes equations, but at this high a Reynolds number all the smallest length and time scales were not resolved. The flow field was solved periodic in the spanwise direction, with spanwise extent $0.142 c$. They used fourth-order compact differences for the vorticity transport equation, except for certain terms which used fourth-order split compact differences for superior short-wave resolution. Fourth-order Runge-kutta explicit time stepping was employed. The grid spacing near the wall yielded: $\Delta x^{+}=27-92, \Delta y^{+}=1.2$, and $\Delta z^{+}=17$. The wall normal jet velocity was specified directly on the body surface. Near the inflow, they tripped the laminar flow to turbulence through the use of a time-harmonic forcing term on the right-hand side of the equations; a high-amplitude 3-D Gaussian shaped disturbance was applied for selected spanwise Fourier components. Overall, they obtained excellent predictions of both mean and turbulence quantities for the baseline and suction conditions, in much better agreement with experiment than typical RANS/URANS results. However, bubble lengths were still somewhat overpredicted. They attributed this overprediction either (1) the need for even wider spanwise domain (they noted significant improvement going from $0.071 c$, used at the CFDVAL2004 workshop, to $0.142 c$ extent), (2) the need to resolve more of the finer scales, or (3) possible differences in freestream turbulence characteristics. Computations were not done for the oscillatory condition. 


\section{Summary and Remaining Challenges}

To summarize the computations for case 1 that have been published since the original CFDVAL2004 workshop, one of the main advancements has been the development/recognition of techniques to better match the boundary conditions of the experiment at the jet exit. When computations matched experiment better at the slot exit, they tended to match better into the field, as would be expected. One method used FFTs and an approximated cavity shape, but others managed to do well by curve fitting or with a better representation of the actual shape. In other words, the cavity shape itself does not appear to be a key factor, but knowledge of the jet exit flow field is needed a priori in these cases in order to iterate to determine appropriate boundary conditions. However, one group managed to simulate the drum-like motion of the piezoelectric membrane in a predictive boundary condition that did not make use of this knowledge at the slot exit. Such a truly predictive method is of course more desirable, because one may then be able to predict actuator performance with only knowledge of the characteristics of the vibrating driver (and no need for taking flow field measurements). Another advancement has been the recognition of the importance of end effects for this flow field. Even with a slot aspect ratio as large as 28, the end effects become significant a relatively short distance from the slot, above approximately $8 h$ or so.

Many different methodologies were seen to be able to yield reasonable results in the near-field, including loworder, URANS, blended RANS-LES, and N-S. For N-S, solely laminar results were generally poor, but with sufficient resolution and small enough numerical dissipation that allowed N-S to develop turbulent structures, results were good in the near-field. Thus, the jet flow field above the slot is characterized by turbulence. But it is unclear at this point whether URANS methods are fully adequate for predicting mean-flow quantities, and if so, which models work and which do not. With high-fidelity boundary conditions, the simple linear SA and SST models appeared to do reasonably well for many features of interest in the near field, but the work of Carpy and Manceau ${ }^{22}$ suggests that linear models are missing key physics and more advanced nonlinear models or even full Reynolds stress model may be required.

Challenges that remain include the need to perform computations that accurately capture the end effects. Can 3-D URANS model these physics, or it is necessary to resolve the turbulent eddies with blended RANS-LES, LES, or DNS? Also, is it possible to use a predictive moving-grid boundary condition similar to that of Xia and Qin ${ }^{23}$ for a 3-D simulation that models the full three-dimensional shape of the actuator, rather than a 2-D extrusion?

To summarize the computations for case 2, there have not been many published results since the CFDVAL2004 workshop. At the workshop it was recognized that both URANS and LES could achieve equally reasonable results in terms of mean flow quantities (both time-averaged and phase-averaged). Including the internal orifice - as opposed to applying a simple periodic surface boundary conditin - was shown to be important for capturing the complex nature of the flow field in its immediate vicinity. Dandois et al. ${ }^{36}$ further clarified the benefits of LES for this type of flow field: it is able to achieve better agreement with turbulence data than URANS. The benefits of appropriate inflow boundary conditions for LES to insure adequate turbulent eddy content was also demonstrated.

Among the challenges that remain: to date no one has used a purely predictive boundary condition to achieve close agreement with the velocity characteristics measured at the orifice exit. However, in light of the still-unexplained large spanwise velocity component in the case 2 data, it may be worth revisiting the experiment or possibly establishing new jet-in-crossflow benchmark experiments for validation.

To summarize the post-workshop computations for case 3, no major progress has been made since the time of the workshop in terms of RANS/URANS. Results have for the most part been very consistent in terms of predicting too little eddy viscosity in the separated region and too long a bubble. It was noted that models can sometimes predict a particular feature like reattachment location correctly for the wrong reasons. For example, because $k-\varepsilon$ turbulence models tend to predict smooth-body separation caused by adverse pressure gradient too late, they also tend to predict earlier reattachment. This reattachment location may appear to agree better with experiment in the hump case, for example, but it is not due to better modeling of the turbulent mixing in the separated region; i.e., the $k-\varepsilon$ physics are still wrong. It has also become clear that good global computations can be obtained for case 3 either by computing the plenum or by specifying some sort of reasonable boundary condition on the body surface. Doing one or the other has not been a determining factor for success or failure. However, it should be stressed that comparisons have not focused on details in the immediate vicinity of the slot.

Blended RANS-LES methods have also been used for case 3 over the last several years, but have met with mixed success. For example, DES does particularly well for the baseline case, but it has had trouble with the suction case because of its smaller separation bubble. DES also sometimes requires that games be played in terms of grid spacing or defining the region where it is to be active.

A note should be made concerning some of the inconsistent trends that have shown up. Most of them have been associated with the use of commercial software. In one case, results consistent with the collective were obtained, but in some other cases the same software produced inconsistent results. On the other hand, most workshop participants 
whose results were consistent with one another were intimately familiar with the CFD software they were using, sometimes even having written it themselves. This should serve as a reminder that CFD software still requires user expertise.

The largest strides have been made for case 3 in the realm of LES, and in one case "coarse-grid" DNS. Using these methods, several groups have computed one or more of the case 3 conditions, obtaining significant improvement over RANS/URANS methods. By resolving many of the 3-D turbulent eddies in the flow field, the mixing due to turbulence in the separated region can be better predicted. A summary of various case 3 reattachment locations - emphasizing the recent blended RANS-LES, LES, and DNS-type results - is given in Table 1. These results are displayed graphically along with the results from the original workshop for the baseline and suction conditions in fig. 19. As mentioned above, the later "coarse-grid" DNS doubled the number of grid points from that used in the workshop by doubling the spanwise extent.

Table 1. Reattachment Locations for Case 3

\begin{tabular}{|c|c|c|c|}
\hline Case & Baseline & Suction & Oscillatory \\
\hline Experiment $^{3,4}$ & $1.11 \pm 0.003$ & $0.94 \pm 0.005$ & $\approx 0.98$ \\
\hline Typical RANS/URANS ${ }^{6,49}$ & 1.24 & 1.10 & 1.22 \\
\hline $\mathrm{DES}^{55}$ & 1.13 & 1.10 & - \\
\hline $\mathrm{DES}^{56}$ & 1.12 & 1.11 & 1.11 \\
\hline $\mathrm{LES}^{56}$ & 1.11 & 0.95 & 1.05 \\
\hline $\mathrm{LES}^{58}$ & 1.09 & 0.95 & 1.01 \\
\hline ILES $^{57}$ & 1.14 & 0.98 & 1.10 \\
\hline “Coarse-grid” DNS ${ }^{60}$ & 1.19 & 1.00 & - \\
\hline
\end{tabular}

Remaining challenges include improving blended RANS-LES methods to work consistently better for this type of flow, especially for cases with smaller separation bubbles. Also, parametric studies using RANS indicated overprediction of separation bubble length even as its size decreased; is there a bubble size small enough for which RANS quantitatively predicts bubble size and physics well? It would also be interesting to see how well LES can predict the trends (due to variations in jet strength, Reynolds number, frequency, etc.) seen in the experiments. Finally, can these expensive simulations be used to help improve RANS turbulence models in the separated region?

CFD has been increasingly called upon to predict time-dependent flow control applications. In order to establish confidence in computational methods for these flows, it is important to achieve a record of documented successes and failures. Workshops such as CFDVAL2004 have helped to shape a part of this record; it is important now to continue to follow through and address the challenges that remain.

\section{Acknowledgments}

The author would like to thank the following people for providing figures and/or data: C.-S. Yao of NASA Langley Research Center, V. N. Vatsa of NASA Langley Research Center, R. Manceau of University of Poitiers/CNRS, S. Carpy of University of Nantes/CNRS, R. Mittal of George Washington University, R. B. Kotapati of Exa Corporation, E. Garnier of ONERA, and D. You of the Center for Turbulence Research. 


\section{References}

${ }^{1}$ Yao, C.-S., Chen, F. J., and Neuhart, D., "Synthetic Jet Flowfield Database for Computational Fluid Dynamics Validation," AIAA Journal, Vol. 44, No. 12, 2006, pp. 3153-3157.

${ }^{2}$ Schaeffler, N. W. and Jenkins, L. N., "Isolated Synthetic Jet in Crossflow: Experimental Protocols for a Validation Dataset," AIAA Journal, Vol. 44, No. 12, 2006, pp. 2846-2856.

${ }^{3}$ Greenblatt, D., Paschal, K. B., Yao, C.-S., Harris, J., Schaeffler, N. W., and Washburn, A. E., "Experimental Investigation of Separation Control Part 1: Baseline and Steady Suction," AIAA Journal, Vol. 44, No. 12, 2006a, pp. 2820-2830.

${ }^{4}$ Greenblatt, D., Paschal, K. B., Yao, C.-S., and Harris, J., "Experimental Investigation of Separation Control Part 2: Zero Mass-Efflux Oscillatory Blowing," AIAA Journal, Vol. 44, No. 12, 2006b, pp. 2831-2845.

${ }^{5}$ Naughton, J. W., Viken, S., and Greenblatt, D., "Skin-Friction Measurements on the NASA Hump Model," AIAA Journal, Vol. 44, No. 6, 2006, pp. 1255-1265.

${ }^{6}$ Rumsey, C. L., Gatski, T. B., Sellers, W. L., III, Vatsa, V. N., and Viken, S. A., "Summary of the 2004 Computational Fluid Dynamics Validation Workshop on Synthetic Jets," AIAA Journal, Vol. 44, No. 2, 2006, pp. 194-207.

${ }^{7}$ Vassberg, J. C., Tinoco, E. N., Mani, M., Brodersen, O. P., Eisfeld, B., Wahls, R. A., Morrison, J. H., Zickuhr, T., Laflin, K. R., Mavriplis, D. J., "Summary of the Third AIAA CFD Drag Prediction Workshop," AIAA Paper 2007-0260, January 2007.

${ }^{8}$ Morrison, J. H. and Hemsch, M. J., "Statistical Analysis of CFD Solutions from the Third AIAA Drag Prediction Workshop," AIAA Paper 2007-0254, January 2007.

${ }^{9}$ Spalart, P. R., and Allmaras, S. R., "A One-Equation Turbulence Model for Aerodynamic Flows," La Recherche Aerospatiale, No. 1, 1994, pp. $5-21$

${ }^{10}$ Favre, A., "Equations des Gaz Turbulents Compressibles," J. Mechanique, Vol. 4, No. 3, 1965, pp. 361-390.

${ }^{11}$ Anderson, D. A., Tannehill, J. C., and Pletcher, R. H., Computational Fluid Mechanics and Heat Transfer, Hemisphere Publishing Corp, Washington, 1984, p. 200.

${ }^{12}$ Hussain, A. K. M. F. and Reynolds, W. C., "The Mechanics of an Organized Wave in Turbulent Shear Flow," Journal of Fluid Mechanics, Vol. 41, 1970, pp. 241-258.

${ }^{13}$ Grinstein, F. F. and Fureby, C., "Recent Progress on MILES for High Reynolds Number Flows," ASME Journal of Fluids Engineering, Vol. 124, December 2002, pp. 848-861.

${ }^{14}$ Piomelli, U., Balaras, E., Squires, K. D., and Spalart, P. R., “Zonal Approaches to Wall-Layer Models for Large-Eddy Simulations,” AIAA Paper 2002-3083, June 2002.

${ }^{15}$ Sagaut, P., Large Eddy Simulation for Incompressible Flows, Third Edition, Springer, Berlin, 2006.

${ }^{16}$ Yamaleev, N. K. and Carpenter, M. H., "Quasi-one-Dimensional Model for Realistic Three-Dimensional Synthetic Jet Actuators," AIAA Journal, Vol. 44, No. 2, February 2006, pp. 208-216.

${ }^{17}$ Vatsa, V. N. and Turkel, E., "Simulation of Synthetic Jets Using Unsteady Reynolds-Averaged Navier-Stokes Equations," AIAA Journal, Vol. 44, No. 2, February 2006, pp. 217-224.

${ }^{18}$ Menter, F. R., "Two-Equation Eddy-Viscosity Turbulence Models for Engineering Applications," AIAA Journal, Vol. 32, No. 8, 1994, pp. $1598-1605$.

${ }^{19}$ Vatsa, V. and Carpenter, M., "Higher-Order Temporal Schemes with Error Controllers for Unsteady Navier-Stokes Equations," AIAA Paper 2005-5245, June 2005.

${ }^{20}$ Zhang, P. F. and Wang, J. J., "Novel Signal Wave Pattern for Efficient Synthetic Jet Generation," AIAA Journal, Vol. 45, No. 5, May 2007, pp. 1058-1065.

${ }^{21}$ Park, S. H., Yu, Y. H., and Byun, D. Y., "RANS Simulations of a Synthetic Jet in Quiescent Air,” AIAA Paper 2007-1131, January 2007.

${ }^{22}$ Carpy, S. and Manceau, R., "Turbulence Modelling of Statistically Periodic Flows: Synthetic Jet into Quiescent Air," Int. Journal of Heat and Fluid Flow, Vol. 27, No. 5, 2006, pp. 756-767.

${ }^{23}$ Xia, H. and Qin, N., "Comparison of Unsteady Laminar and DES Solutions of Synthetic Jet Flow," AIAA Paper 2006-3161, June 2006.

${ }^{24}$ Spalart, P. R., Jou, W.-H., Strelets, M., and Allmaras, S. R., "Comments on the Feasibility of LES for Wings, and on a Hybrid RANS/LES," Prodeedings of 1st AFOSR International Conference on DNS and LES, Louisiana Tech., Greyden Press, 1997. pp. 137-147.

${ }^{25}$ Cui, J. and Agarwal, R. K., "Three-Dimensional Computation of a Synthetic Jet in Quiescent Air," AIAA Journal, Vol. 44, No. 12, December 2006, pp. 2857-2865.

${ }^{26}$ Mani, M. and Bush, R. H., "A Two-Equation Large Eddy Stress Model for High Sub-Grid Shear," AIAA Paper 2001-2561, 2001.

${ }^{27}$ Kotapati, R. B., Mittal, R., and Cattafesta, L. N. III, “Numerical Study of a Transitional Synthetic Jet in Quiescent External Flow,” Journal of Fluid Mechanics, Vol. 581, 2007, pp. 287-321.

${ }^{28}$ Iaccarino, G., Marongiu, C., Catalano, P., and Amato, M., "RANS Modeling and Simulations of Synthetic Jets," AIAA Paper 2004-2223, June-July 2004.

${ }^{29}$ Durbin, P. A., "Separated Flow Computations with the $k-\varepsilon-v^{2}$ Model," AIAA Journal, Vol. 33, No. 4, 1995, pp. 659-664.

${ }^{30}$ Biedron, R., Vatsa, V., and Atkins, H., "Simulation of Unsteady Flows Using an Unstructured Navier-Stokes Solver on Moving and Stationary Grids," AIAA Paper 2005-5093, June 2005.

${ }^{31}$ Rumsey, C. L., "Computation of a Synthetic Jet in a Turbulent Cross-Flow Boundary Layer," NASA/TM-2004-213273, October 2004.

${ }^{32}$ Rumsey, C. L. and Gatski, T. B., "Summary of EASM Turbulence Models in CFL3D with Validation Test Cases," NASA/TM-2003-212431, June 2003.

${ }^{33}$ Rumsey, C. L., Schaeffler, N. W., Milanovic, I. M., and Zaman, K. B. M. Q., "Time-Accurate Computations of Isolated Circular Synthetic Jets in Crossflow," Computers \& Fluids, Vol. 36, No. 6, 2007, pp. 1092-1105.

${ }^{34}$ Cui, J. and Agarwal, R. K., "3-D CFD Validation of an Axisymmetric Jet in Cross-Flow (NASA Langley Workshop Validation: Case 2)”, AIAA Paper 2005-1112, January 2005.

${ }^{35}$ Xia, H., Qin, N., "Detached-Eddy Simulation for Synthetic Jets with Moving Boundaries," Modern Physics Letters B, Vol. 19, Nos. 28-29, 2005, pp. 1429-1434. 
${ }^{36}$ Dandois, J., Garnier, E., and Sagaut, P., "Unsteady Simulation of Synthetic Jet in a Crossflow," AIAA Journal, Vol. 44, No. 2, February 2006, pp. $225-238$

${ }^{37}$ Seifert, A. and Pack, L. G., "Active Flow Separation Control on Wall-Mounted Hump at High Reynolds Numbers," AIAA Journal, Vol. 40, No. 7, 2002, pp. 1363-1372.

${ }^{38}$ Johansson, T. G. and Davidson, L., “11th ERCOFTAC Workshop on Refined Turbulence Modelling,” ERCOFTAC Bulletin, Vol. 69, 2006, pp. $28-34$.

${ }^{39}$ Thiele, F. and Jakirlic, S., "12th ERCOFTAC/IAHR/COST Workshop on Refined Turbulence Modelling," ERCOFTAC Bulletin, Vol. 75, December 2007, pp. 5-10.

${ }^{40}$ Leschziner, M. A., "Test Case 9.2: The Flow in a Channel with Periodic Hills on One Wall," Proceedings of the 10th Joint ERCOFTAC (SIG-15) / IAHR / QNET-CFD Workshop on Refined Turbulence Modelling, Ed. R. Manceau, J.-P. Bonnet, M. A. Leschziner, F. Menter, CNRS / Universite de Poitiers / ENSMA, France, October 2002.

${ }^{41}$ Rumsey, C. L., "Effect of Turbulence Models on Two Massively-Separated Benchmark Flow Cases," NASA/TM-2003-212412, May 2003.

${ }^{42}$ Capizzano, F., Catalano, P., Marongiu, C., and Vitagliano, P. L., "U-RANS Modelling of Turbulent Flows Controlled by Synthetic Jets," AIAA Paper 2005-5015, June 2005.

${ }^{43}$ Cui, J. and Agarwal, R. K., "CFD Validation of Turbulent Separation Control on a 2D Hump (NASA Langley Workshop Validation: Case 3)," AIAA Paper 2005-5013, June 2005.

${ }^{44}$ Balakumar, P., "Computations of Flow over a Hump Model Using Higher Order Method with Turbulence Modeling," AIAA Paper 20051270, January 2005.

${ }^{45}$ Morgan, P. E., Rizzetta, D. P., and Visbal, M. R., "High-Order Numerical Simulation of Turbulent Flow over a Wall-Mounted Hump," AIAA Journal, Vol. 44, No. 2, February 2006, pp. 239-251.

${ }^{46}$ Bettini, C. and Cravero, C., "Computational Analysis of Flow Separation Control for the Flow Over a Wall-Mounted Hump Using a Synthetic Jet," AIAA Paper 2007-0516, January 2007.

${ }^{47}$ He, C., Corke, T. C., and Patel, M. P., "Numerical and Experimental Analysis of Plasma Flow Control Over a Hump Model," AIAA Paper 2007-0935, January 2007.

${ }^{48}$ Madugundi, D., Nagib, H., and Kiedaisch, J., "Evaluation of Turbulence Models Through Prediction of Separated Flows with and without Flow Control and Circulation Effects," AIAA Paler 2008-0567, January 2008.

${ }^{49}$ Rumsey, C., "Reynolds-Averaged Navier-Stokes Analysis of Zero Efflux Flow Control over a Hump Model," Journal of Aircraft, Vol. 44 , No. 2, 2007, pp. 444-452.

${ }^{50}$ Rumsey, C. L. and Greenblatt, D., "Parametric Study of Flow Control Over a Hump Model Using an Unsteady Reynolds-Averaged NavierStokes Code," NASA/TM-2007-214897, September 2007.

${ }^{51}$ Israel, D. M., Postl, D., and Fasel, H. F., "A Flow Simulation Methodology for Analysis of Coherent Structures and Flow Control," AIAA Paper 2004-2225, June-July 2004.

${ }^{52}$ Hiller, S. J. and Seitz, P. A., "The Interaction Between a Fluidic Actuator and Main Flow Using SAS Turbulence Modeling," AIAA Paper 2006-3678, June 2006.

${ }^{53}$ Menter, F. R. and Egorov, Y., “A Scale-Adaptive Simulation Model Using Two-Equation Models," AIAA Paper 2005-1095, January 2005.

${ }^{54}$ Biswas, D., "Studies on Separation Control CFD Validation Test Case Based on a Higher Order LES Model," AIAA Paper 2006-3684, June 2006.

${ }^{55}$ Krishnan, V., Squires, K. D., and Forsythe, J. R., "Prediction of Separated Flow Characteristics over a Hump,” AIAA Journal, Vol. 44, No. 2 , February 2006, pp. 252-262

${ }^{56}$ Saric, S., Jakirlic, S., Djugum, A., and Tropea, C., "Computational Analysis of a Locally Forced Flow over a Wall-Mounted Hump at High-Re Number," Int. Journal of Heat and Fluid Flow, Vol. 27, No. 4, 2006, pp. 707-720.

${ }^{57}$ Morgan, P. E., Rizzetta, D. P., Visbal, M. R., "Large-Eddy Simulation of Separation Control for Flow Over a Wall-Mounted Hump," AIAA Journal, Vol. 45, No. 11, November 2007, pp. 2643-2660.

${ }^{58}$ You, D., Wang, M., and Moin, P., "Large-Eddy Simulation of Flow over a Wall-Mounted Hump with Separation Control," AIAA Journal, Vol. 44, No. 11, November 2006, pp. 2571-2577.

${ }^{59}$ Franck, J. A. and Colonius, T., "Large-Eddy Simulation of Separation Control for Compressible Flow over a Wall-Mounted Hump," AIAA Paper 2008-0555, January 2008.

${ }^{60}$ Postl, D. and Fasel, H. F., "Direct Numerical Simulation of Turbulent Flow Separation from a Wall-Mounted Hump," AIAA Journal, Vol. 44, No. 2, February 2006, pp. 263-272.

${ }^{61}$ Saric, S., Jakirlic, S., and Tropea, C., "Computational Analysis of a Locally Forced Flow over a Wall-Mounted Hump at High-Re Number," Fouthh International Symposium on Turbulence and Shear Flow Phenomena, ed. J. A. C. Humphrey, J. K. Eaton, R. Friedrich, N. Kasagi, M. A. Leschziner, T. B. Gatski, June 2005, pp. 1189-1194.

${ }^{62}$ Morgan, P. E., Rizzetta, D. P., Visbal, M. R., "Large-Eddy Simulation of Separation Control for Flow Over a Wall-Mounted Hump," AIAA Paper 2005-5017, June 2005.

${ }^{63}$ Krishnan, V., Squires, K. D., and Forsythe, J. R., "Prediction of Separated Flow Characteristics over a Hump Using RANS and DES," AIAA Paper 2004-2224, June 2004. 


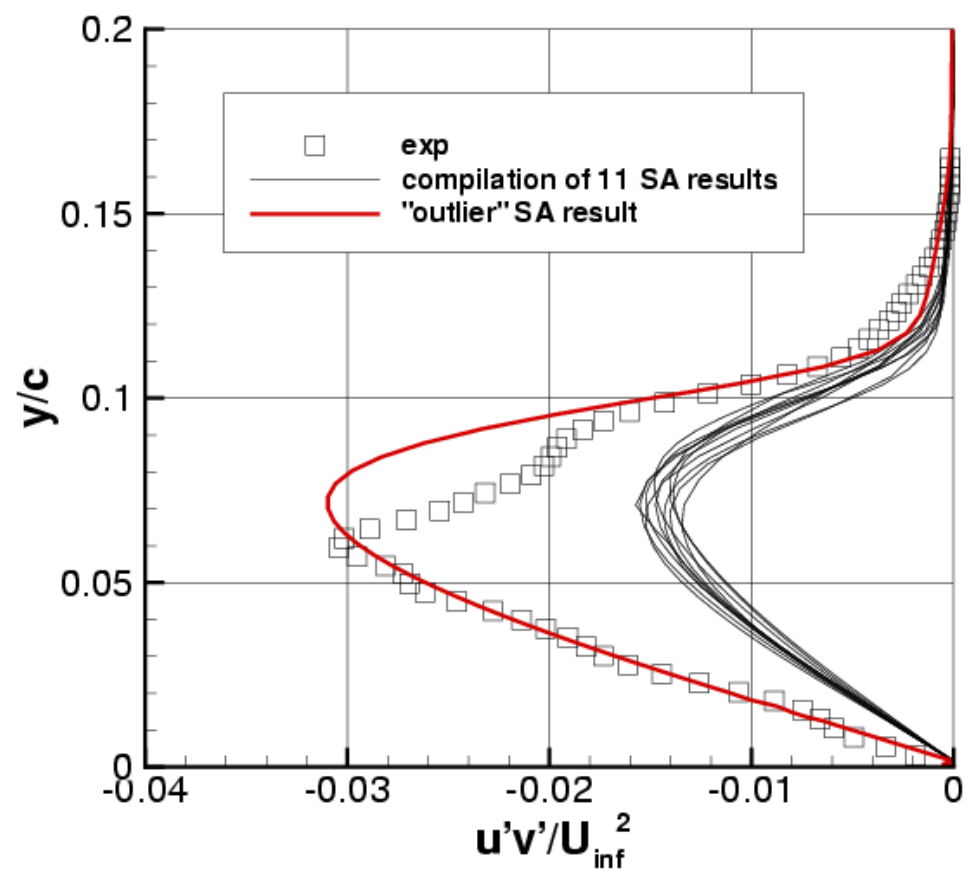

Figure 1. Turbulent shear stresses from CFDVAL2004 workshop case 3 in separated region of hump model, using SA turbulence model in various codes for a variety of 2-D structured and unstructured grids.

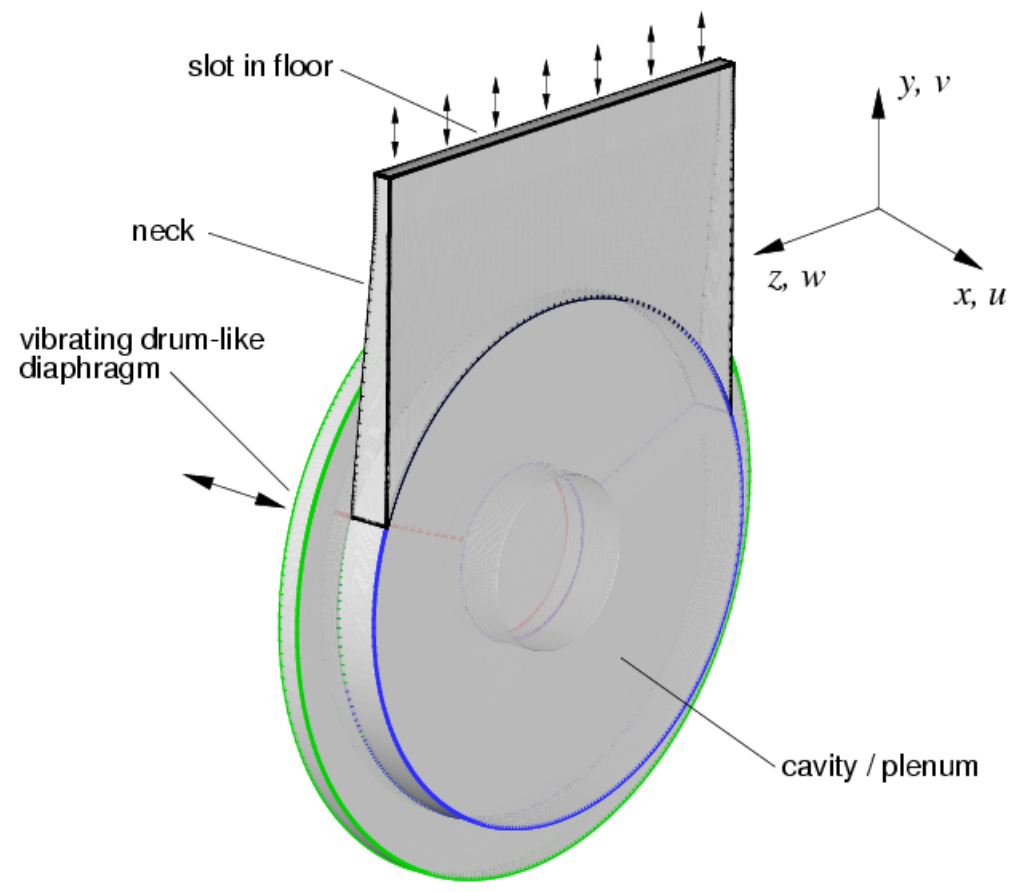

Figure 2. Diagram showing case 13-D slot and plenum configuration, with circular piezoelectric driver on far side of view. 


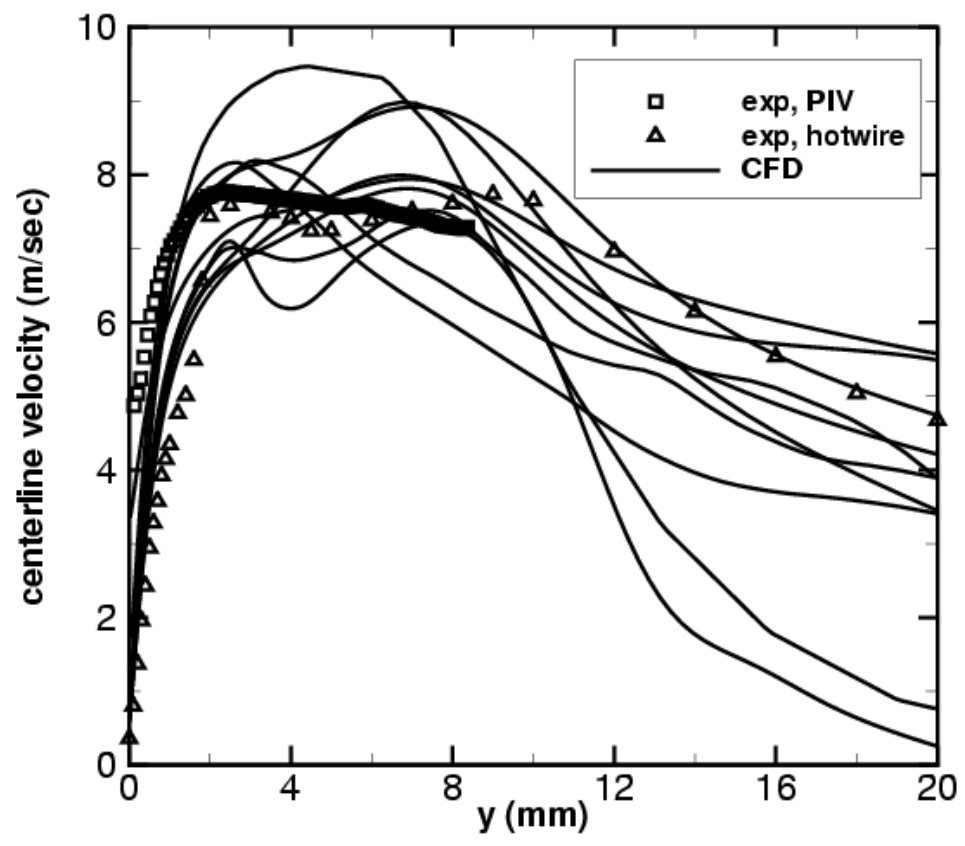

Figure 3. Time-averaged vertical velocity along the jet centerline for case 1, showing representative results from 10 different workshop participants, along with original experiment.
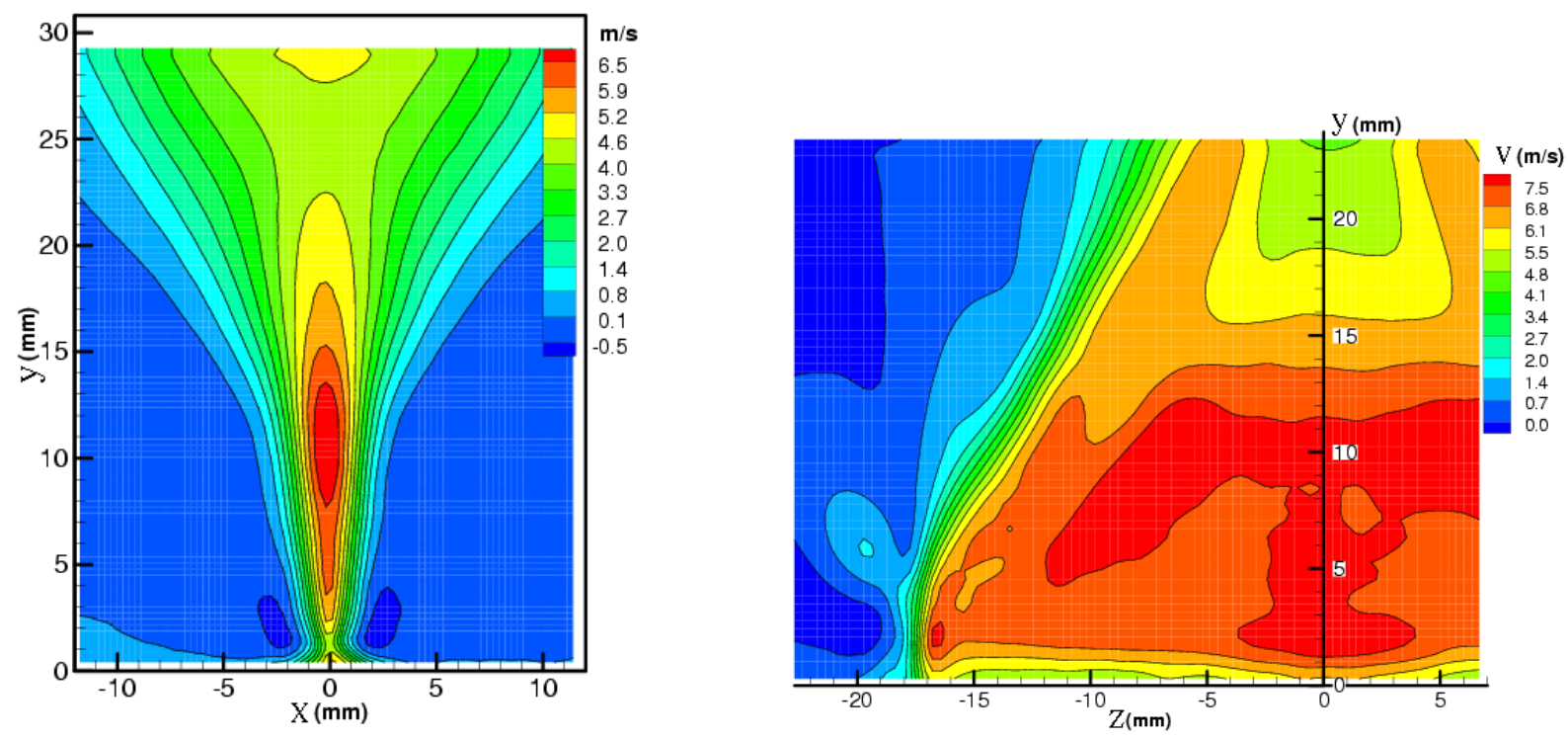

Figure 4. Contours of case 1 mean jet velocity from PIV experimental data of Yao et al. ${ }^{1}$ (a) across the slot, and (b) along the slot. 


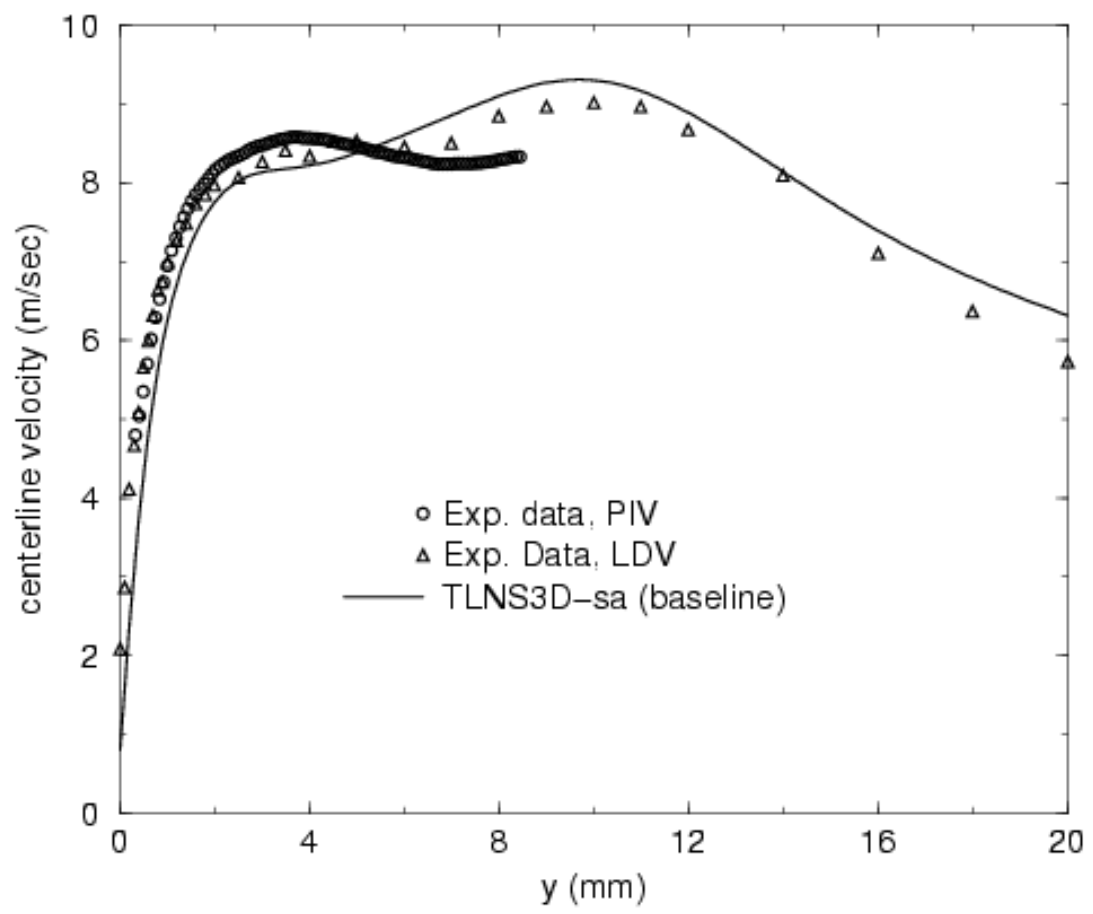

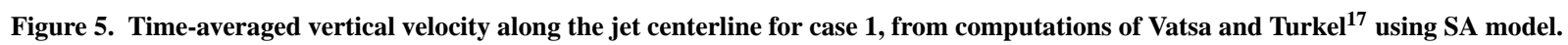

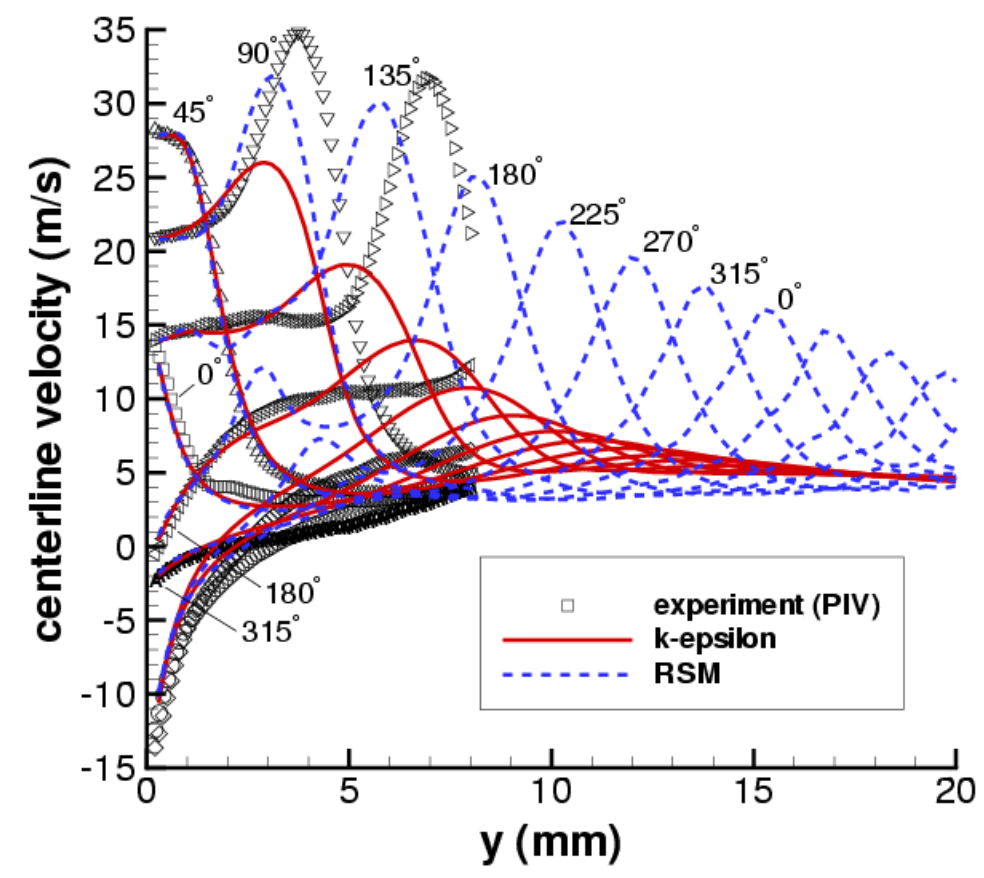

Figure 6. Effect of two different turbulence models on the vertical phase-averaged velocity profiles for case 1 in the symmetry plane, from Carpy and Manceau. ${ }^{22}$ 


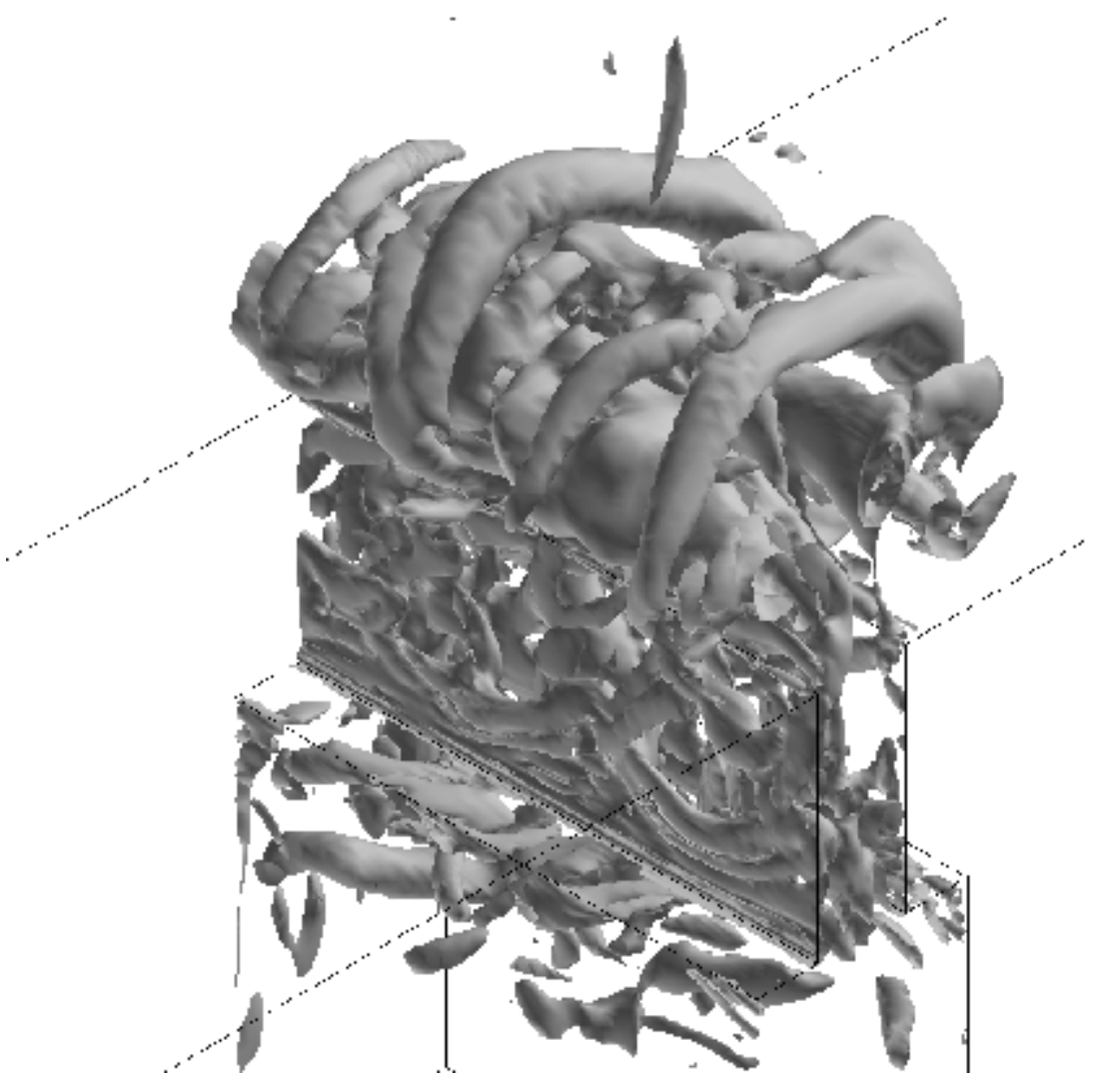

Figure 7. Visualization of vortical structures in the near field of case 1 from N-S computations of Kotapati et al. ${ }^{27}$

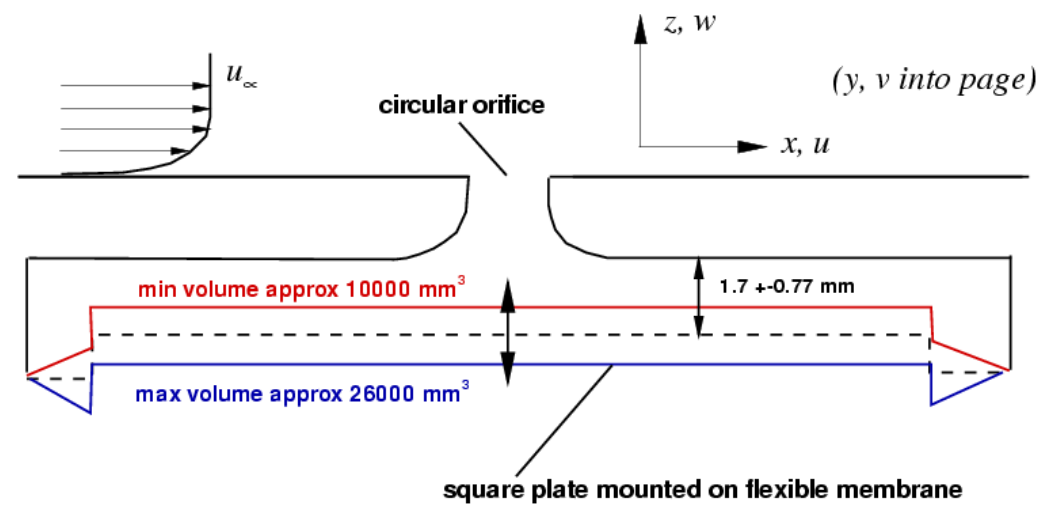

Figure 8. Sketch of case 2 plenum and orifice. 

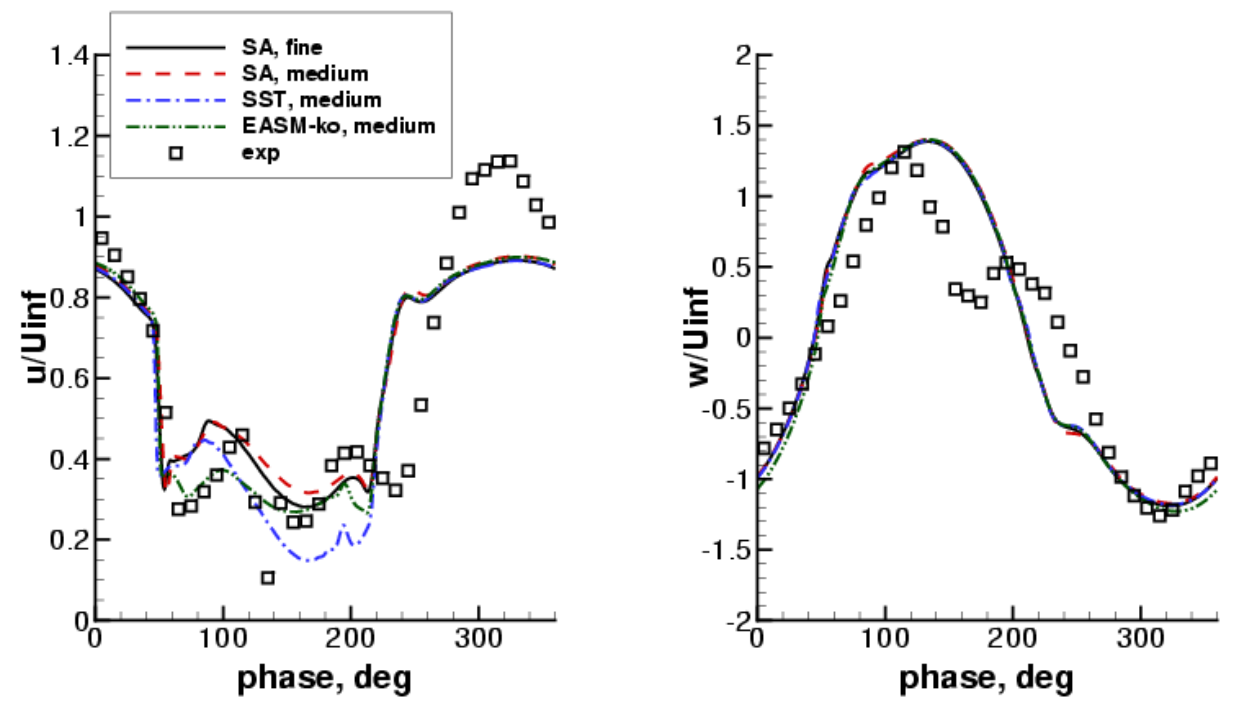

Figure 9. Time histories of velocity components for case 2 over center of the orifice; (a) $u$-velocity (streamwise), (b) $w$-velocity (vertical).
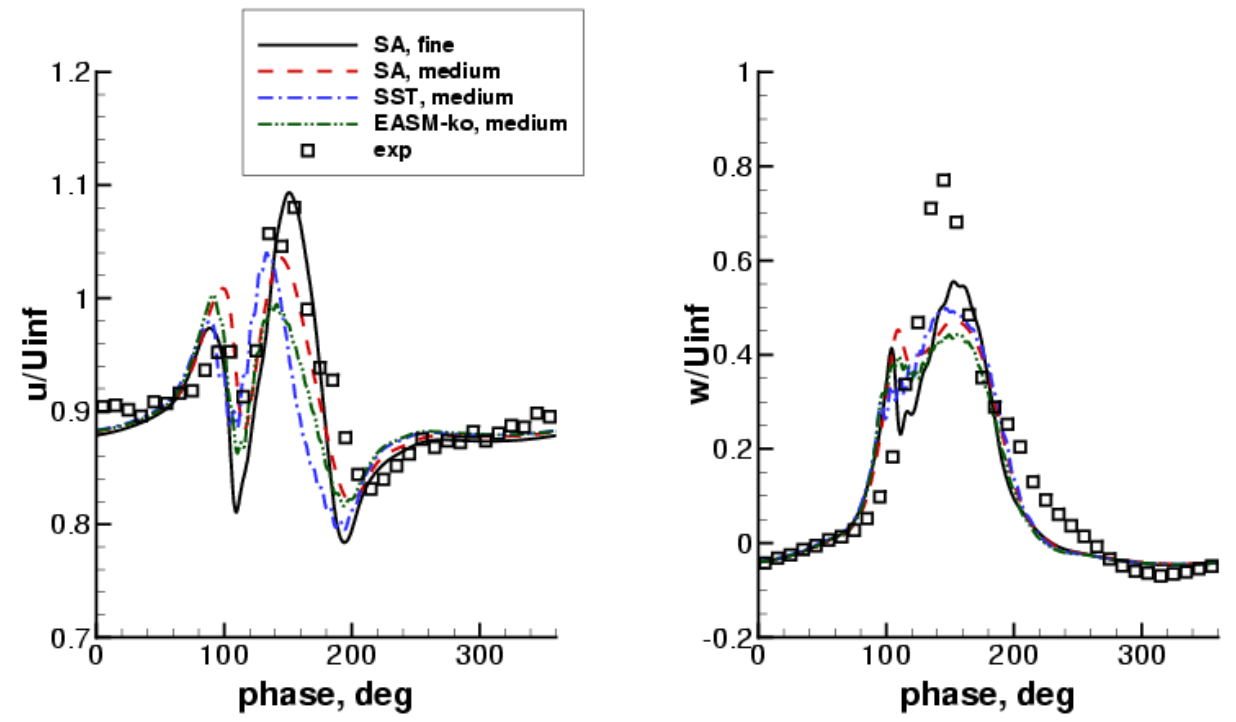

Figure 10. Time histories of case 2 velocity components 1 diameter downstream at center-plane, at $z=10 \mathrm{~mm}$ above surface; (a) $u$-velocity (streamwise), (b) $w$-velocity (vertical). 

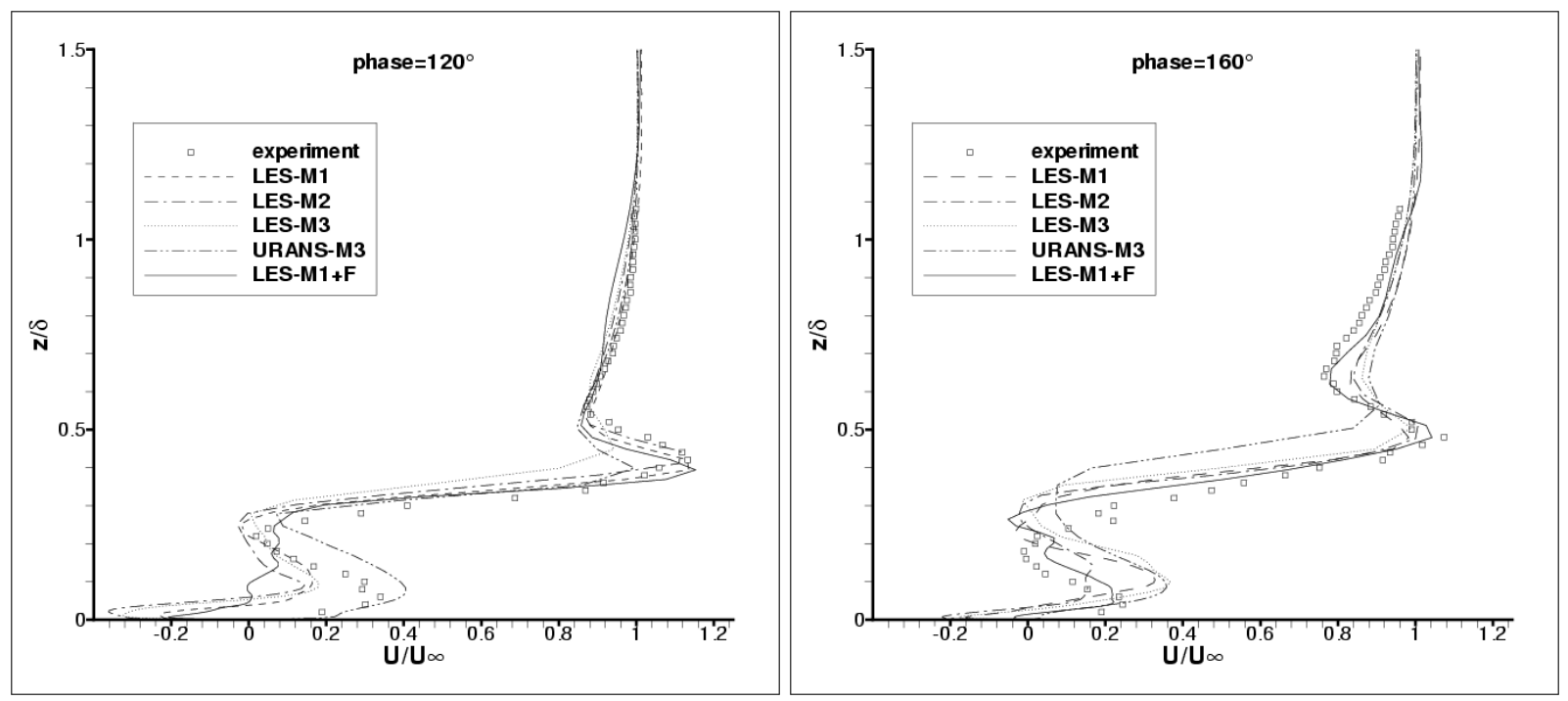

Figure 11. Phase-averaged case $2 u$-velocity 1 diameter downstream at center-plane; (a) phase $120^{\circ}$, (b) phase $160^{\circ}$. Figures from Dandois et al. ${ }^{36}$ Used with permission.
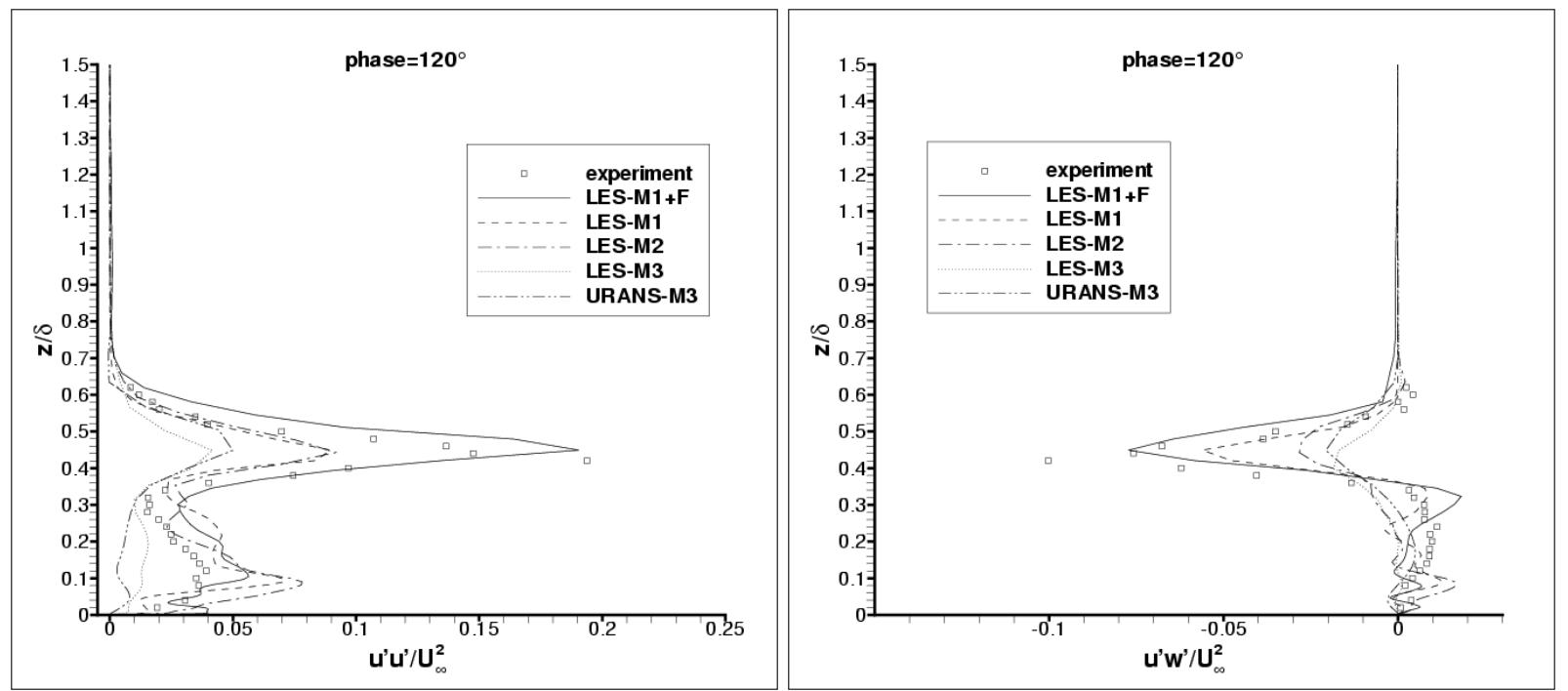

Figure 12. Phase-averaged case 2 normal and shear stresses 1 diameter downstream at center-plane at phase $120^{\circ}$; (a) $u^{\prime} u^{\prime} / U_{\infty}^{2},($ b) $u^{\prime} w^{\prime} / U_{\infty}^{2}$. Figures from Dandois et al. ${ }^{36}$ Used with permission. 


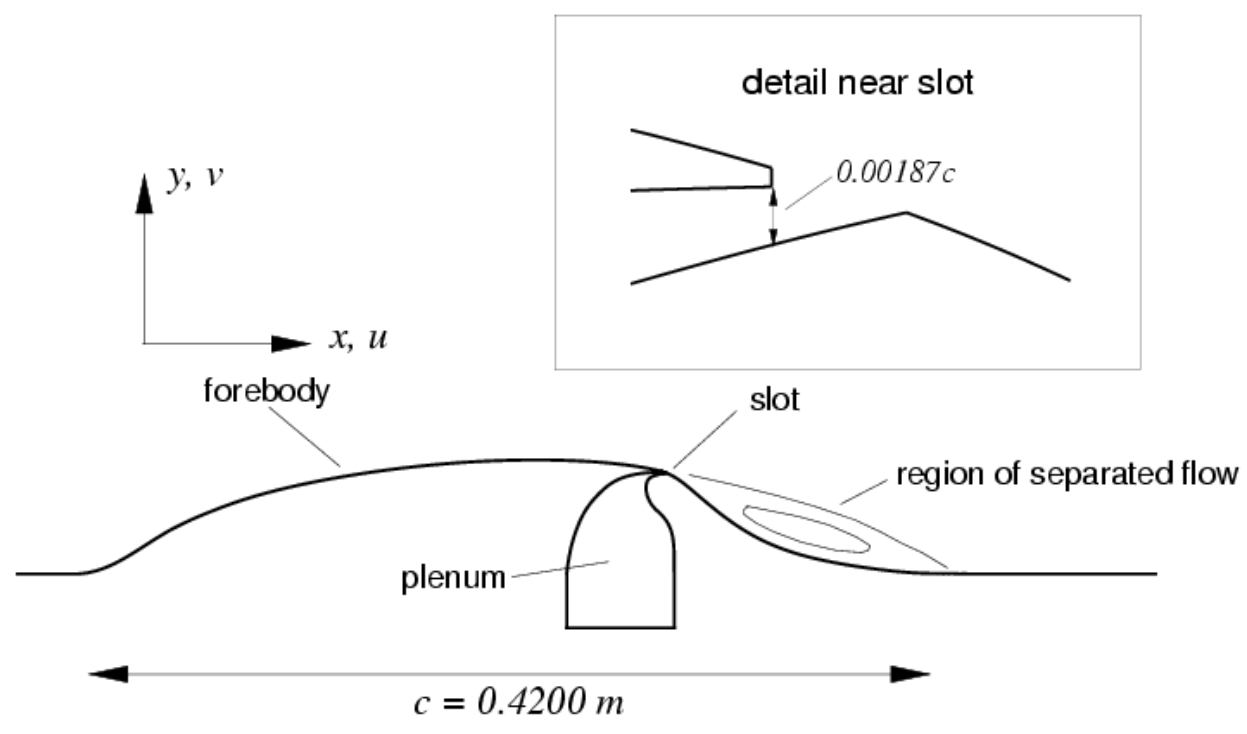

Figure 13. Sketch of case 3 hump model.

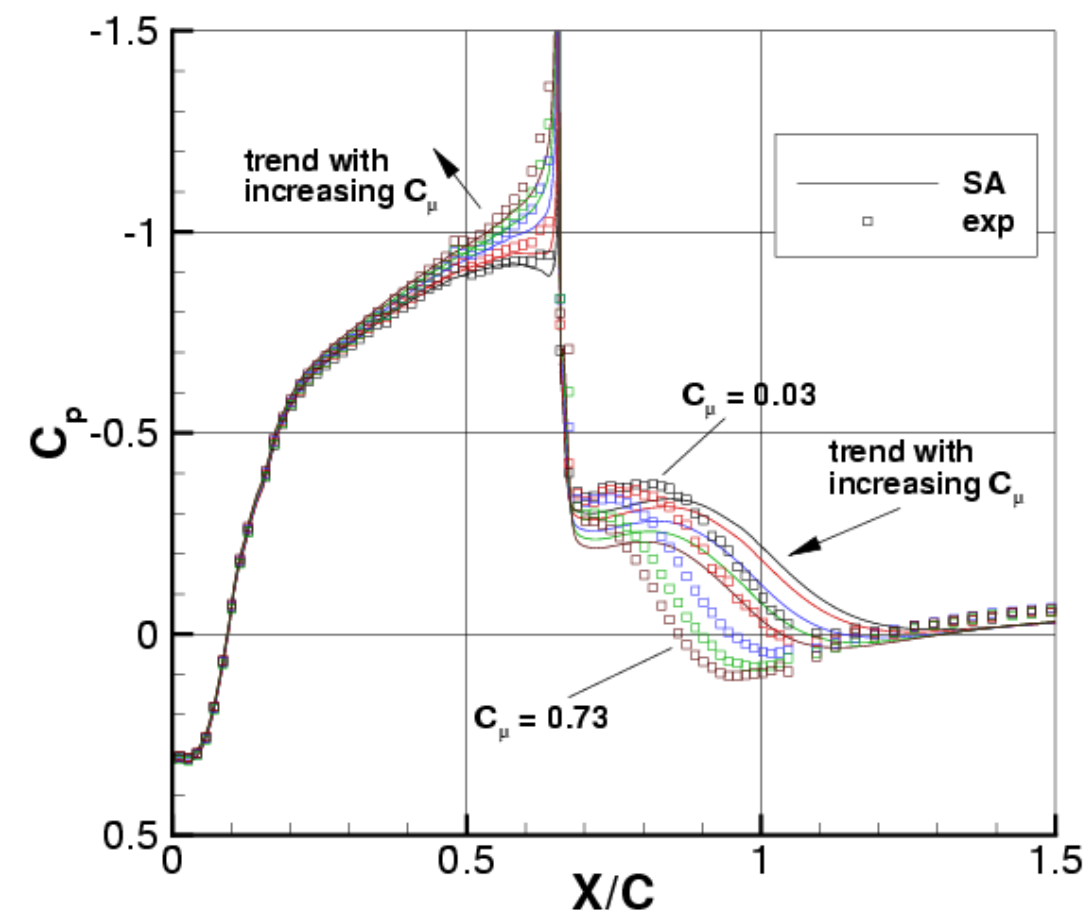

Figure 14. Effect of $C_{\mu}$ on surface pressure coefficients for steady suction on hump model at $R e=0.936$ million, $\mathbf{S A}$ model. 


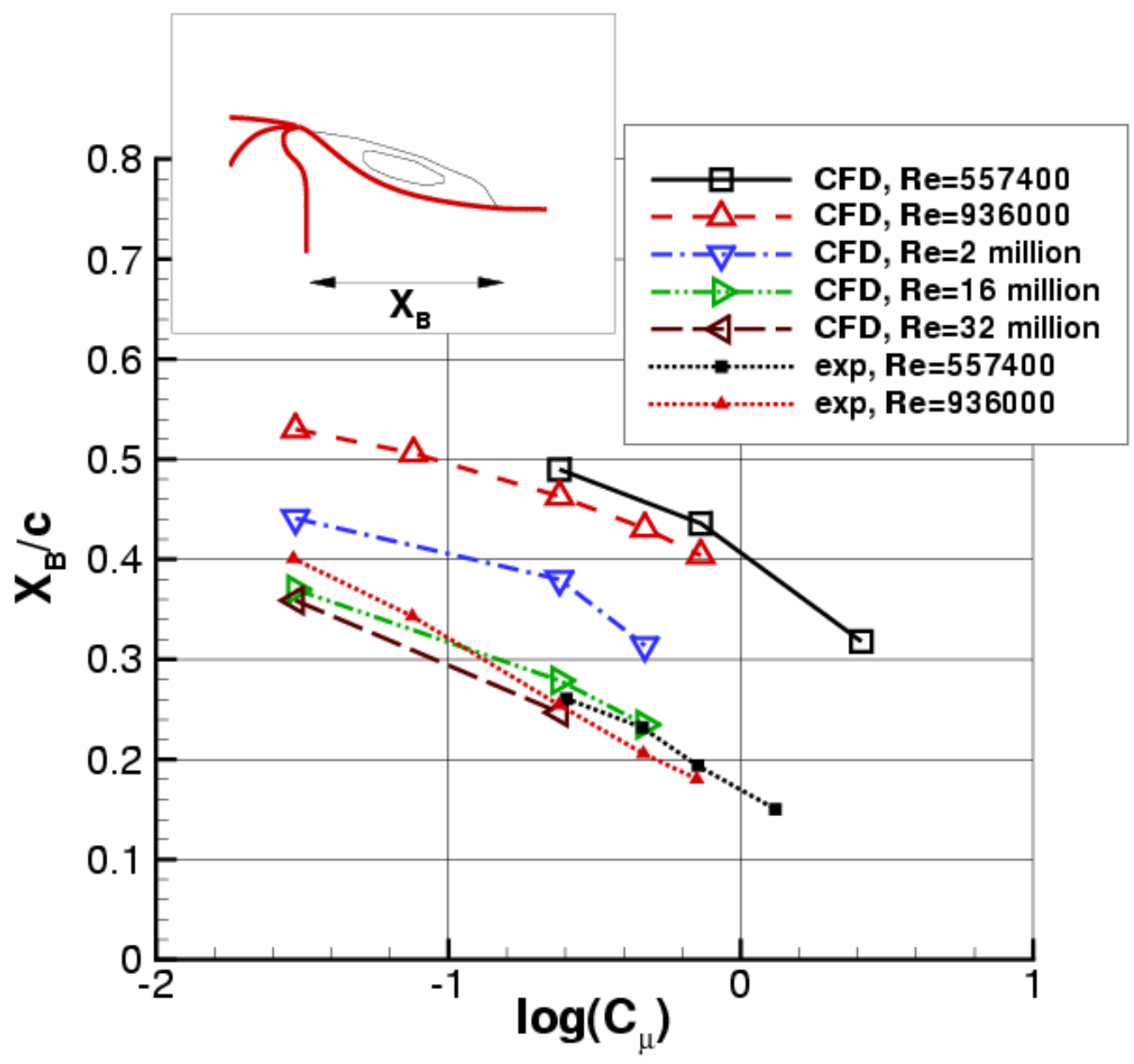

Figure 15. Bubble length as a function of $C_{\mu}$ and Reynolds number for steady suction, SA model. 

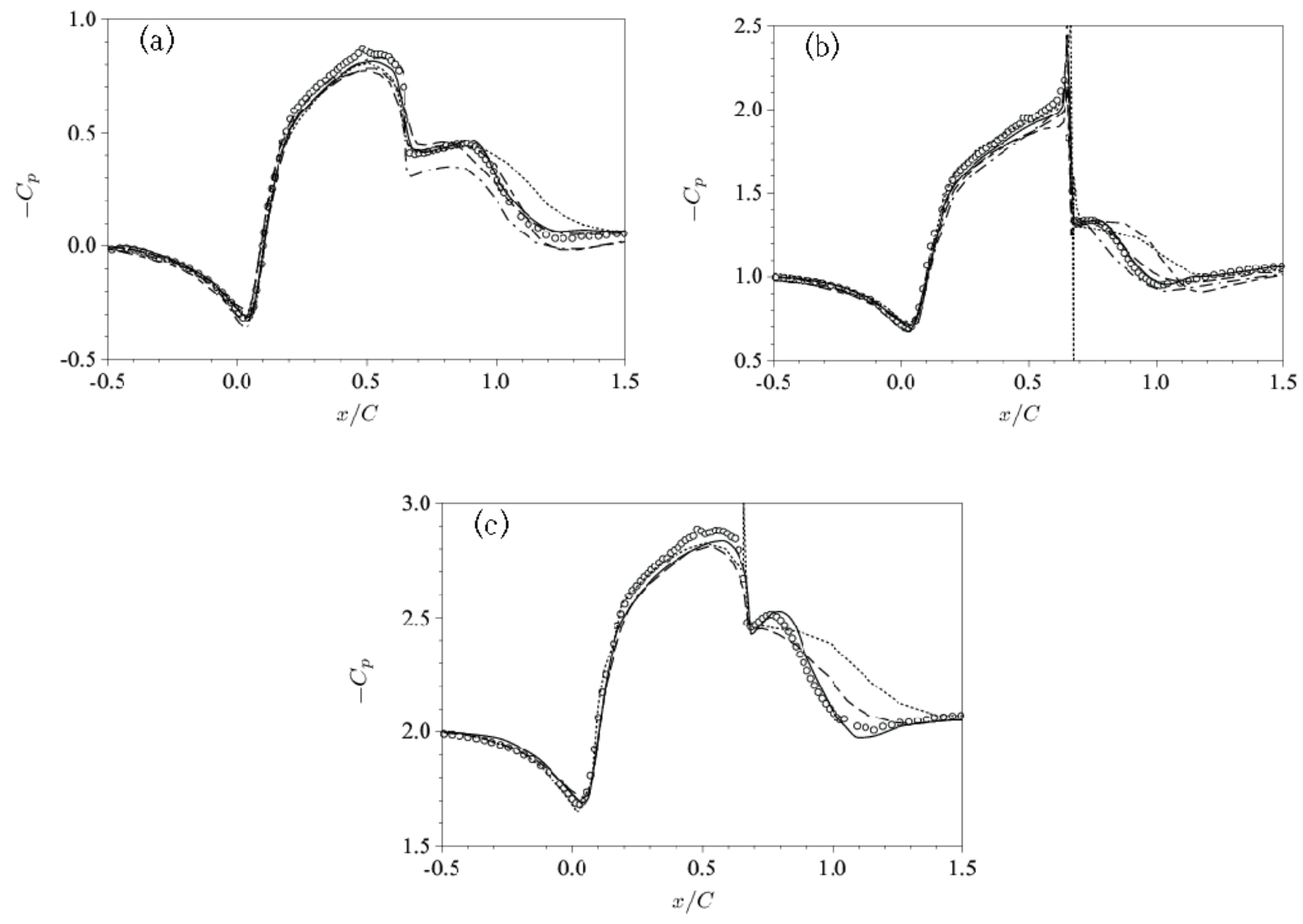

Figure 16. Surface pressure coefficient for case 3 (a) baseline (no flow control), (b) steady suction, (c) oscillatory control. Solid line: LES with dynamic model ${ }^{58}$ dash-dotted line: LES with constant-coefficient model; $;{ }^{61}$ dashed line: ILES $;{ }^{62}$ dash-short dash line: DES; ${ }^{63}$ dotted line: URANS; ${ }^{42}$ circles: experiment. ${ }^{3,4}$ Figures from You et al. ${ }^{58}$ Used with permission.

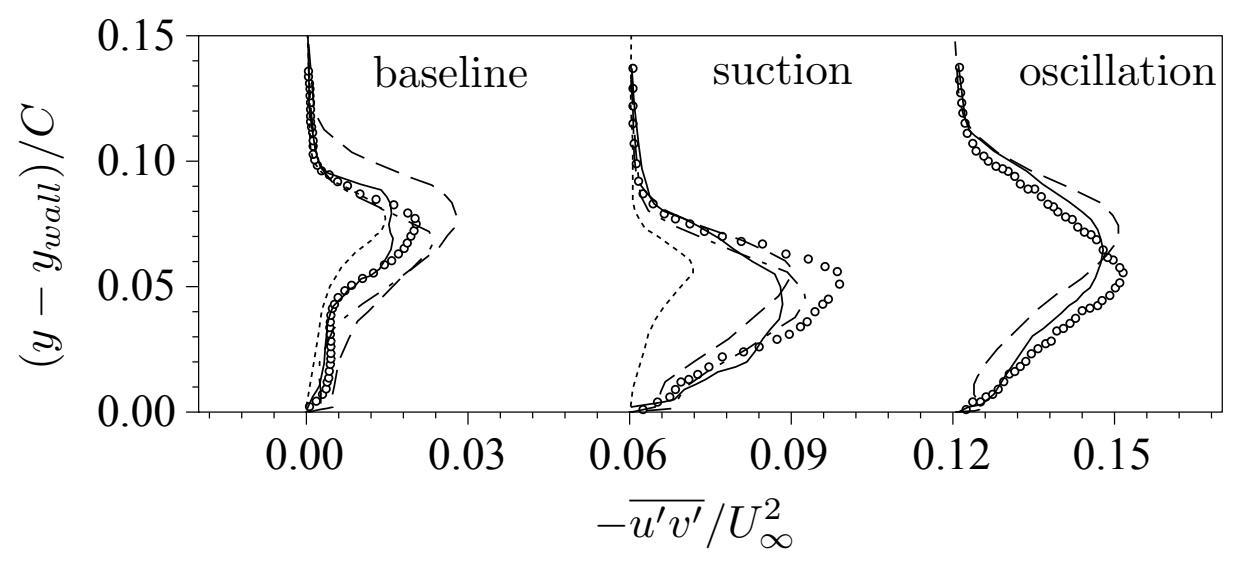

Figure 17. Turbulent shear stress profiles for case 3 at $x / c=0.8$ (in separated region). Solid line: LES with dynamic model; ${ }^{58}$ dash-dotted line: LES with constant-coefficient model; ${ }^{61}$ dashed line: ILES; ${ }^{62}$ dotted line: URANS; ${ }^{42}$ circles: experiment. $^{3,4}$ Figure from You et al. $^{58}$ Used with permission. 
(a)

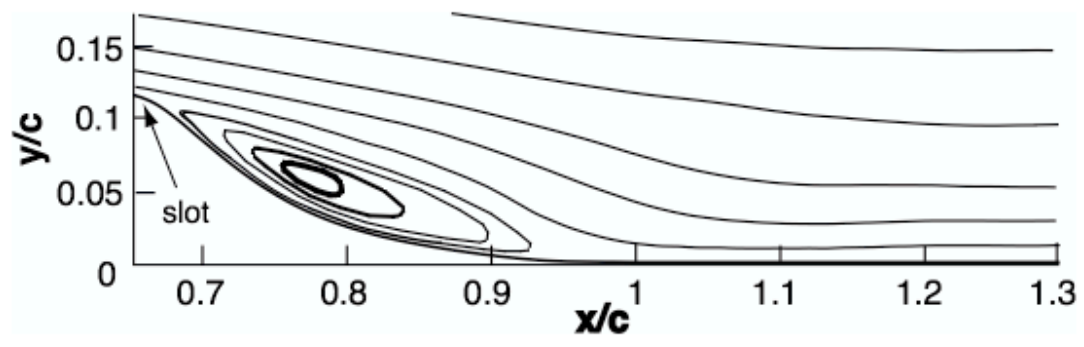

(b)

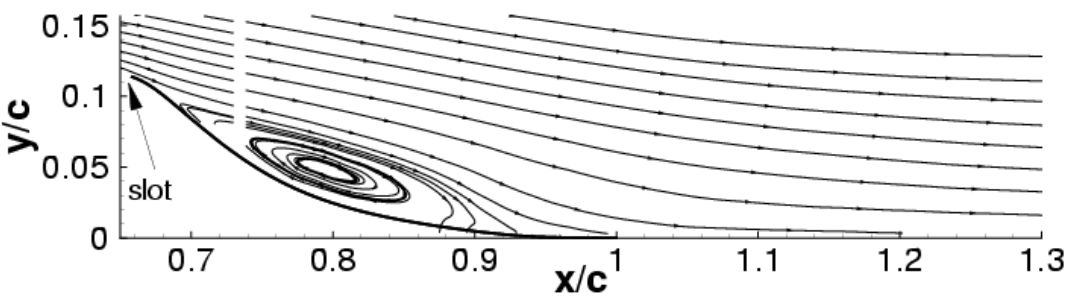

Figure 18. Streamlines for case 3 with steady suction; (a) LES computations of You et al., ${ }^{58}$ (b) experiment.
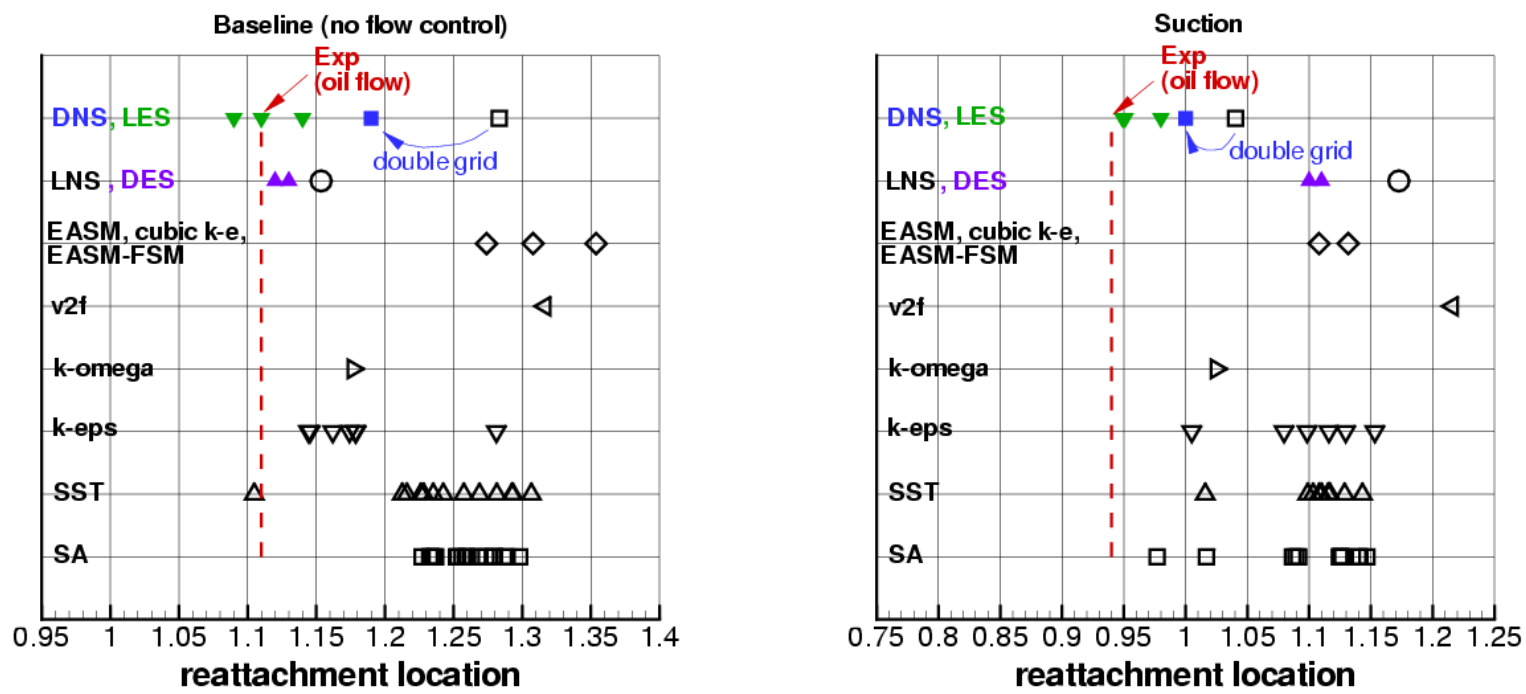

Figure 19. Reattachment locations from original workshop (open black symbols) along with more recent DES (filled violet delta symbols), LES (filled green gradient symbols), and "coarse-grid” DNS (filled blue square symbol), for (a) baseline condition, (b) suction condition. 